The Astrophysical Journal, 647:1040-1055, 2006 August 20

(C) 2006. The American Astronomical Society. All rights reserved. Printed in U.S.A.

\title{
MINKOWSKI'S OBJECT: A STARBURST TRIGGERED BY A RADIO JET, REVISITED
}

\author{
Steve Croft, ${ }^{1,2}$ Wil van Breugel, ${ }^{1,2}$ Wim de Vries, ${ }^{1,3}$ Mike Dopita, ${ }^{4}$ Chris Martin, ${ }^{5}$ Raffaella Morganti, ${ }^{6,7}$ \\ Susan Neff, ${ }^{8}$ Tom Oosterloo, ${ }^{6,7}$ David Schiminovich, ${ }^{9}$ S. A. Stanford, ${ }^{1,3}$ and Jaceueline van Gorkom ${ }^{9}$ \\ Received 2006 February 4; accepted 2006 April 26
}

\begin{abstract}
We present neutral hydrogen, ultraviolet, optical, and near-infrared imaging, and optical spectroscopy, of Minkowski's Object (MO), a star-forming peculiar galaxy near NGC 541. The observations strengthen evidence that star formation in MO was triggered by the radio jet from NGC 541 . Key new results are the discovery of a $4.9 \times 10^{8} M_{\odot}$ double $\mathrm{H}_{\text {I }}$ cloud straddling the radio jet downstream from MO, where the jet changes direction and decollimates; strong detections of $\mathrm{MO}$, also showing double structure, in $\mathrm{UV}$ and $\mathrm{H} \alpha$; and numerous $\mathrm{H}$ II regions and associated clusters in $\mathrm{MO}$. In $\mathrm{UV}$, MO resembles the radio-aligned, rest-frame UV morphologies in many high-redshift radio galaxies (HzRGs), also thought to be caused by jet-induced star formation. MO's stellar population is dominated by a $7.5 \mathrm{Myr}$ old, $1.9 \times 10^{7} M_{\odot}$ instantaneous burst, with a current star formation rate of $0.52 M_{\odot} \mathrm{yr}^{-1}$ (concentrated upstream from where the $\mathrm{H} \mathrm{I}$ column density is high). This is unlike the jet-induced star formation in Centaurus A, where the jet interacts with preexisting cold gas; in MO, the $\mathrm{H}$ I may have cooled out of a warmer, clumpy intergalactic or interstellar medium as a result of jet interaction, followed by the collapse of the cooling clouds and subsequent star formation (consistent with numerical simulations). Since the radio source that triggered star formation in MO is much less luminous, and therefore more common than powerful HzRGs, and because the environment around MO is not particularly special in terms of abundant dense, cold gas, jet-induced star formation in the early universe might be even more prevalent than previously thought.
\end{abstract}

Subject headings: galaxies: jets — galaxies: starburst — stars: formation

\section{INTRODUCTION}

In a previous paper, 20 years ago, van Breugel et al. (1985) hypothesized that the starburst seen in the peculiar "Minkowski's Object" (MO; Minkowski 1958), in the cluster Abell 194, was triggered by the radio jet emerging from the nucleus of the nearby active galaxy NGC 541. Only one other example of jet-induced star formation was known at the time, in Centaurus A (Graham \& Price 1981; Brodie et al. 1983), and the phenomenon of jetinduced star formation was considered more of a curiosity than something worthy of general interest. In both cases, the radio galaxies are at low redshift and of relatively low radio luminosity. Subsequent observations, with more sensitive detectors, larger telescopes, and better spatial resolution, have shown that jetinduced star formation is more common than initially thought, and examples have now been found in sources at low as well as high redshift, and with low (FR I type) or high (FR II type) radio luminosity (Fanaroff \& Riley 1974).

For example, we now know that star formation in low-redshift, low radio luminosity radio galaxies can occur in a variety of en-

\footnotetext{
${ }^{1}$ Institute of Geophysics and Planetary Physics, Lawrence Livermore National Laboratory L-413, 7000 East Avenue, Livermore, CA 94550; scroft@ igpp.ucllnl.org.

${ }^{2}$ University of California, Merced, P.O. Box 2039, Merced, CA 95344.

${ }^{3}$ University of California, Davis, 1 Shields Avenue, Davis, CA 95616.

${ }^{4}$ Research School of Astronomy and Astrophysics, Australian National University, Cotter Road, Weston Creek, ACT 2611, Australia.

${ }^{5}$ California Institute of Technology, Pasadena, CA 91125.

${ }^{6}$ Netherlands Foundation for Research in Astronomy, Postbus 2, 7990 AA Dwingeloo, Netherlands.

7 Kapteyn Astronomical Institute, University of Groningen, P.O. Box 800, 9700 AV Groningen, Netherlands.

${ }^{8}$ NASA Goddard Space Flight Center, Laboratory for Astronomy and Solar Physics, Code 681, Greenbelt, MD 20771.

9 Department of Astronomy, Columbia University, 538 West 120th Street, New York, NY 10027.
}

vironments such as the central intergalactic medium (IGM) in X-ray clusters like Abell 1795 (McNamara 2002; O'Dea et al. 2004) and the interstellar medium (ISM) of their parent galaxies (e.g., Wills et al. 2004). Some of the most dramatic jetinduced star formation, with star formation rates (SFR) as high as $1000 M_{\odot} \mathrm{yr}^{-1}$, is associated with very luminous radio galaxies at redshifts up to $z \sim 4$ (Dey et al. 1997; Bicknell et al. 2000). In all these cases, the radio sources are embedded in relatively dense and presumably clumpy media, as evidenced by X-ray halos, cold gas ( $\mathrm{H}$ I), dust, bright line emission, or a combination of these. Theoretical considerations suggest that the star formation may be induced by moderately slow $\left(<1000 \mathrm{~km} \mathrm{~s}^{-1}\right)$ radiative shocks as they encounter IGM or ISM clouds (De Young 1989; Rees 1989). These qualitative models have been developed primarily for the powerful high-redshift radio galaxies, where the star formation is triggered by sideways expanding, radiative shocks in the relatively dense IGM/ISM of the gaseous halos in protoclusters or young parent galaxies (Bicknell et al. 2000; Klamer et al. 2004).

However, little is known with certainty of the astrophysical conditions within the radio jets, including the composition of the jet fluid and its speed, as well as their environment, especially at high redshift. Better knowledge about this would be of great general interest, as it would help with modeling outflows from black hole accretion disks and the conditions of the ISM/IGM in forming galaxies and their protoclusters. Numerical simulations are being used to explore the physical parameter range that is thought to be relevant to gain further insight. MO is of special interest to help constrain such simulations, since there is no obvious indication that the jet is propagating through some especially dense IGM or ISM environment. The Abell 194 cluster does not have a luminous "cooling" X-ray halo like Abell 1795, and there is no evidence for much cold gas outside the jet like in Centaurus A. Thus a better understanding of how the star formation in $\mathrm{MO}$ 
was triggered by the jet would also help in determining how general a phenomenon this might be.

So why did the NGC 541 radio jet trigger star formation? Was it just a chance encounter with a gas-rich galaxy that wandered into the path of the radio jet, which was then "rejuvenated"? This would have been a lucky shot, given that MO is the only peculiar system within the central $\sim 1 \mathrm{Mpc}$ of Abell 194, and that its total (projected) diameter is the same as that of the radio jet. However, if there were an old stellar population underlying the starburst in MO, then this could not be ruled out. On the other hand, as the observations of Abell 1795 have shown, the interaction of jets with warm, clumpy gas in cooling flow clusters may well trigger the collapse of IGM clouds and subsequent star formation.

The development of the new, massively parallel COSMOS numerical simulations package (Anninos et al. 2003) at Livermore allowed Fragile et al. (2004) to simulate the collision of an FR I type jet with a gas cloud, in an effort to see whether star formation could be induced by the jet in the case of MO. They assumed that NGC 541 is surrounded by a multiphase medium resembling galaxy cluster atmospheres (e.g., Ferland et al. 2002); the simulations began with a hot "mother" cloud with a semimajor axis of $10 \mathrm{kpc}$, a semiminor axis of $5 \mathrm{kpc}$, density $0.1 \mathrm{~cm}^{-3}$, and temperature $10^{6} \mathrm{~K}$. Within the mother cloud, denser, warm clouds were assumed to be embedded with typical sizes of $100 \mathrm{pc}$, densities of $10 \mathrm{~cm}^{-3}$, and temperatures of $10^{4} \mathrm{~K}$. Studies of the prototypical FR I radio galaxy 3C 31 (Laing \& Bridle 2002) were used to estimate plausible parameters for the jet at the location of MO, $15 \mathrm{kpc}$ from NGC 541: a velocity of $9 \times 10^{4} \mathrm{~km} \mathrm{~s}^{-1}$, a density of $10^{-4} \mathrm{~cm}^{-3}$, and a temperature of $10^{9} \mathrm{~K}$. Fragile et al. (2004) concluded that it was indeed plausible that such star formation could be triggered by the driving of a radiative shock into such a cloud, causing the cloudlets to compress, radiatively cool, and break up into numerous dense, cold fragments. These fragments survive for many dynamical timescales and are presumably precursors to star formation.

Motivated by these results from numerical simulations, and by the availability of new observing facilities since the previous study, we find this an opportune time to revisit MO in an attempt to better understand the nature of jet-cloud interactions. We first discuss the various new observations obtained, supplemented by archival observations. Then we proceed with an analysis of the spectroscopic and spectral energy distribution information, a discussion of the kinematics, morphology, and timescales, and finally, our conclusions and ideas for future work. We assume an $\Omega_{m}=0.27, \Omega_{\Lambda}=0.73, H_{0}=71 \mathrm{~km} \mathrm{~s}^{-1} \mathrm{Mpc}^{-1}$ cosmology (Spergel et al. 2003). At $z=0.019, \mathrm{MO}$ and the Abell 194 cluster are both at a comoving radial distance of $78.7 \mathrm{Mpc}$, and have a linear scale size of $0.374 \mathrm{kpc} \operatorname{arcsec}^{-1}$.

\section{OBSERVATIONS}

\subsection{VLA Radio Continuum Data}

Van Breugel et al. (1985) published 1.4 GHz Very Large Array (VLA) maps (taken in the A, B, and C array) of MO. ${ }^{10}$ These original radio data were rereduced for the present investigation, using standard routines in the AIPS software package. This was done in order to benefit from the cumulative improvements to the software over the years since 1985. This new radio continuum image has a resolution of $3^{\prime \prime}$, and is included in Figures 1

\footnotetext{
${ }^{10}$ The VLA is operated by the National Radio Astronomy Observatory, which is a facility of the National Science Foundation, operated under cooperative agreement by Associated Universities, Inc.
}

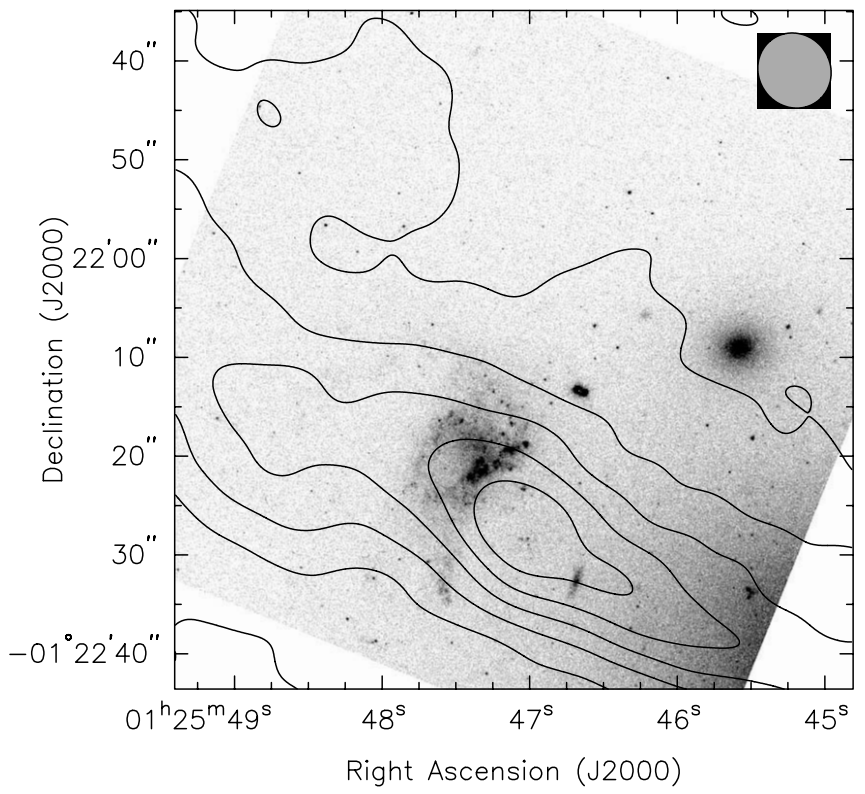

FIG. 1.-Co-added HST F555W+F814W image of MO, overlaid with radio continuum contours from the VLA. Contours are from 0 to $10 \mathrm{mJy}^{\mathrm{J}}$ beam ${ }^{-1}$ at intervals of $2 \mathrm{mJy}$ beam ${ }^{-1}$. The gray ellipse represents the $7.8 \times 77^{\prime \prime} 3$ CLEAN beam $\left(\mathrm{PA}=36^{\circ}\right)$ of the radio observations.

and 2. We refer to the original paper for more details on the radio data.

\subsection{HST Archival $V$ - and I-Band WFPC2 Images}

Although not the main target, MO was within the field of two short (460 s each) Hubble Space Telescope (HST) WFPC2/ F555W and WFPC2/F814W observations of NGC 541 (Verdoes Kleijn et al. 1999), which are available in the archives. These are the highest spatial resolution images of MO, with a measured FWHM of $00^{\prime \prime} 17$ at F555W and $0^{\prime \prime} 20$ at F814W.

MO shows considerable structure with a total projected size that is approximately the same as the jet diameter (Fig. 1). The largest and brightest component (hereafter referred to as MONorth) is found along the northern edge of the jet. Its brightest portion, a $3 \mathrm{kpc}-$ long barlike feature, lies on the side closest to NGC 541 and extends at almost a right angle to the jet. A much fainter, linear feature (hereafter referred to as MO-South) extends to the south across the jet. The central, high surface brightness portion of the radio jet bisects these two components just south of the position of the peak of the star formation as seen in the optical images (Fig. 1). Apart from the bar, MO-North contains several filamentary structures downstream in the jet, and is dotted with compact (mostly unresolved at the $\sim 75 \mathrm{pc}$ resolution of $H S T$ ) young star clusters and $\mathrm{H}$ II regions.

\subsection{Lick Wide Field R-Band Imaging}

To investigate the local environment of $\mathrm{MO}$, the central portion of the Abell 194 cluster was imaged by Michael Gregg on 2002 October 6, using the Prime Focus Camera (PFCam) on the Lick Observatory Shane $3 \mathrm{~m}$ telescope. Eight $600 \mathrm{~s}$ exposures were made using the Spinrad $R$ filter, and five $600 \mathrm{~s}$ exposures were made in the $B$ band. The frames were reduced in the standard manner in IRAF, ${ }^{11}$ and co-added using offsets determined from individual astrometric solutions for each frame. The

11 IRAF is distributed by the National Optical Astronomy Observatories, which are operated by the Association of Universities for Research in Astronomy, Inc., under cooperative agreement with the National Science Foundation. 


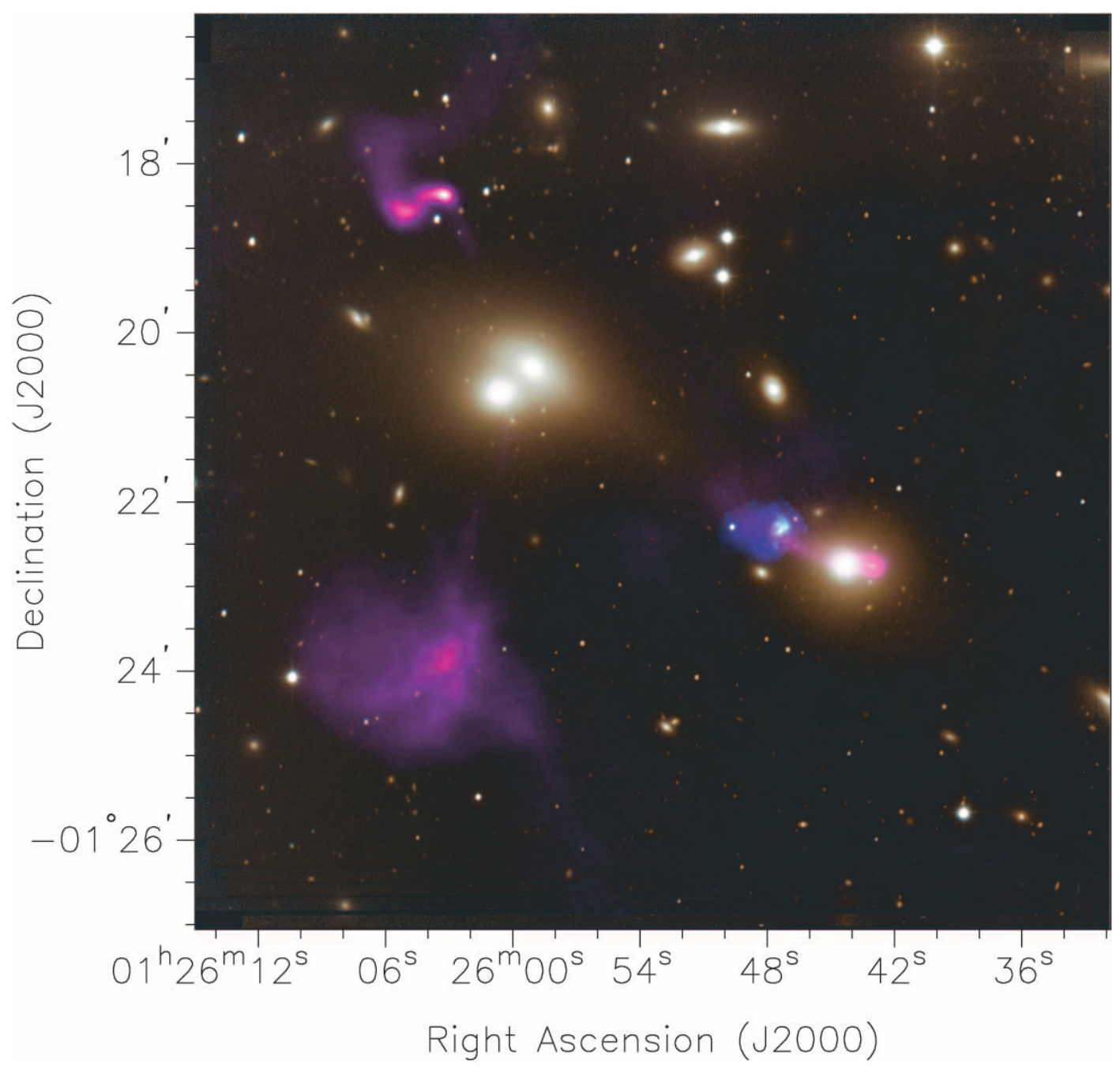

Fig. 2.-Co-added PFCam (BR) image of the field of Abell 194 (color), overlaid with radio continuum (purple), H I data (dark blue), and H $\alpha$ data (light blue). Minkowski's Object is clearly seen as the peculiar object at $1^{\mathrm{h}} 25^{\mathrm{m}} 47^{\mathrm{s}},-1^{\circ} 22^{\prime} 20^{\prime \prime}$, forming from the cloud of $\mathrm{H}_{\mathrm{I}}$ in the path of the jet from NGC $541\left(1^{\mathrm{h}} 25^{\mathrm{m}} 44^{\mathrm{s}}\right.$, $\left.-1^{\circ} 22^{\prime} 46^{\prime \prime}\right)$. The radio galaxy to the left of the image is $3 \mathrm{C} 40\left(1^{\mathrm{h}} 26^{\mathrm{m}} 00^{\mathrm{s}},-01^{\circ} 20^{\prime} 43^{\prime \prime}\right)$, associated with NGC 547 . The galaxy at $1^{\mathrm{h}} 25^{\mathrm{m}} 59^{\mathrm{s}},-01^{\circ} 20^{\prime} 25^{\prime \prime}$, interacting with NGC 547, is NGC 545 .

measured FWHM in the final image is 1".5, and the total field of view is $11^{\prime} \times 11^{\prime}$ (Figs. 2 and 3 ).

The image shows a large $(\sim 20 \times 45 \mathrm{kpc})$ stellar bridge connecting the ellipticals NGC 541 and the interacting galaxies NGC 545/547, which are associated with the large FR I radio source 3C 40 (Simkin 1976). MO is located in this bridge, at least as seen in projection (Fig. 4), opening the possibility that the gas associated with MO has had its origin in some previous tidal interaction between these galaxies. The redshifts of these various systems are consistent: $z=0.0181$ (NGC 541; Smith et al. 2000), $z=0.0189$ (MO; this paper), $z=0.0178$ (NGC 545; Smith et al. 2000), and $z=0.0182$ (NGC 547; Trager et al. 2000).

\subsection{Narrow-Band $\mathrm{H} \alpha$ Imaging}

To determine the total extent of the star formation in $\mathrm{MO}$ and search for other possible emission-line features, we obtained a narrowband, dithered $\mathrm{H} \alpha$ image on 2004 December 17 using "Imager" on the $2.3 \mathrm{~m}$ Advanced Technology Telescope (ATT) at Siding Spring Observatory. Three $200 \mathrm{~s}$ exposures of MO were made in the $R$-band filter, and three $600 \mathrm{~s}$ exposures in a narrowband filter centered at $668 \mathrm{~nm}$ (to match redshifted $\mathrm{H} \alpha$ in $\mathrm{MO})$. Three $30 \mathrm{~s}$ exposures in $R$ band were made of the emissionline flux standard 205-26.7 (Dopita \& Hua 1997), and three $100 \mathrm{~s}$ exposures in a narrowband filter centered at $658 \mathrm{~nm}$ to provide the $\mathrm{H} \alpha$ flux calibration. The images in each filter were reduced in the standard manner in IRAF, using sky flat-fields appropriate to each filter. Once the individual images had been registered and coadded, an astrometric fit was performed for each coadd, and the broadband images were registered and geometrically scaled so that the pixel positions of objects in the broadband and narrowband images matched to subarcsecond accuracy.

Continuum subtraction was performed using the technique of Böker et al. (1999). Briefly, the reduced, registered broadband and narrowband images were rebinned to a size of $135 \times 135$ pixels (each pixel representing 256 pixels of the original image). The intensity (in $\mathrm{DN} \mathrm{s}^{-1}$ ) of each pixel in the rebinned $R$-band image was then plotted against that of the same pixel in the rebinned narrowband image, excluding the brightest 500 pixels in the narrowband image. A linear, least-squares fit was performed, the slope of which represents the scaling factor between the two filters. The unbinned $R$-band image was then convolved with a Gaussian filter to match the PSF of the narrowband image. It was then scaled by the slope of the fit obtained above, and subtracted from the unbinned narrowband image.

The scaling obtained for the standard star observations was 0.0438 , while that for the MO observations was 0.0554 . This is 


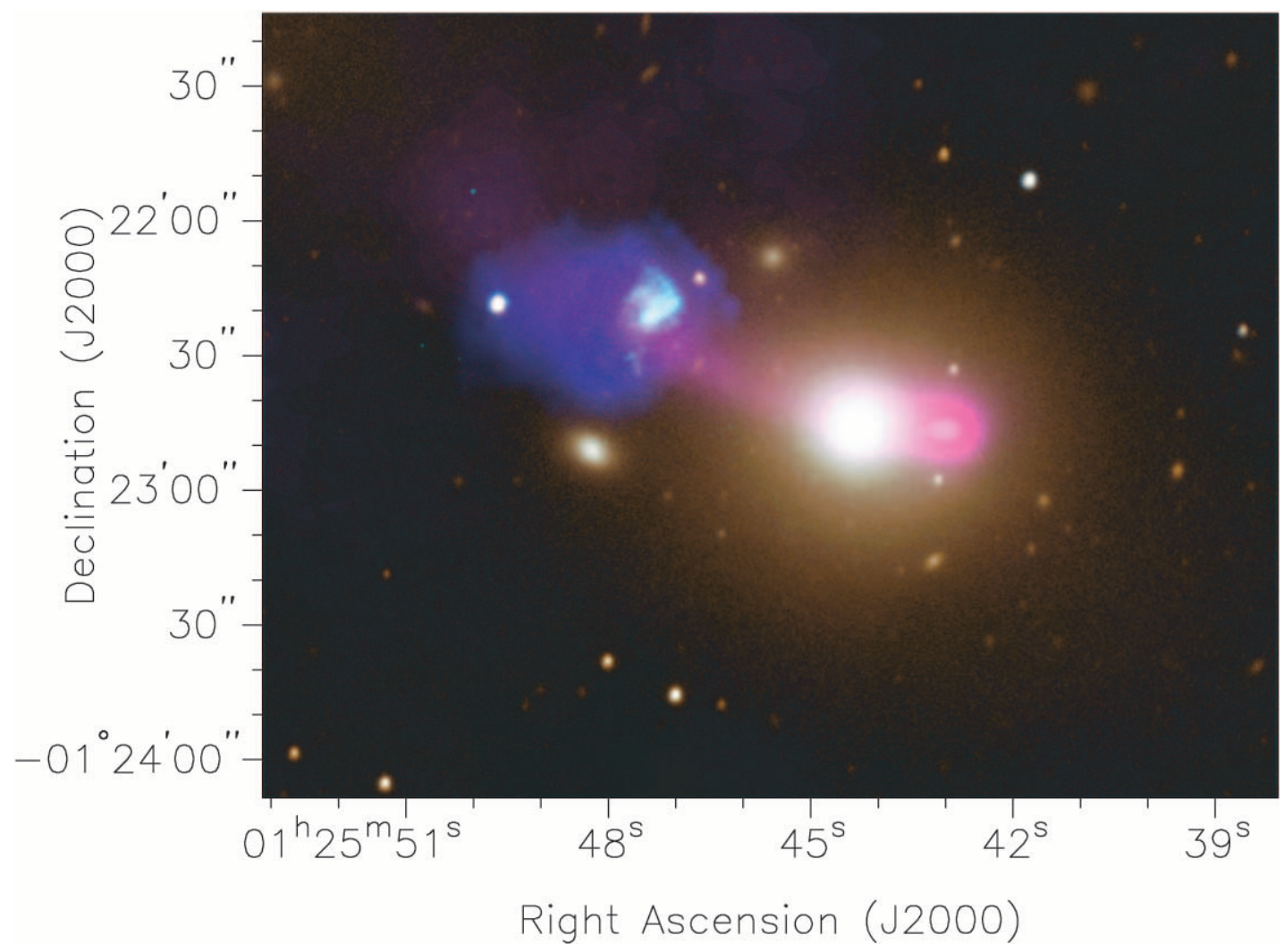

FIG. 3.-Zoom of Fig. 2 to show in more detail the region surrounding MO.

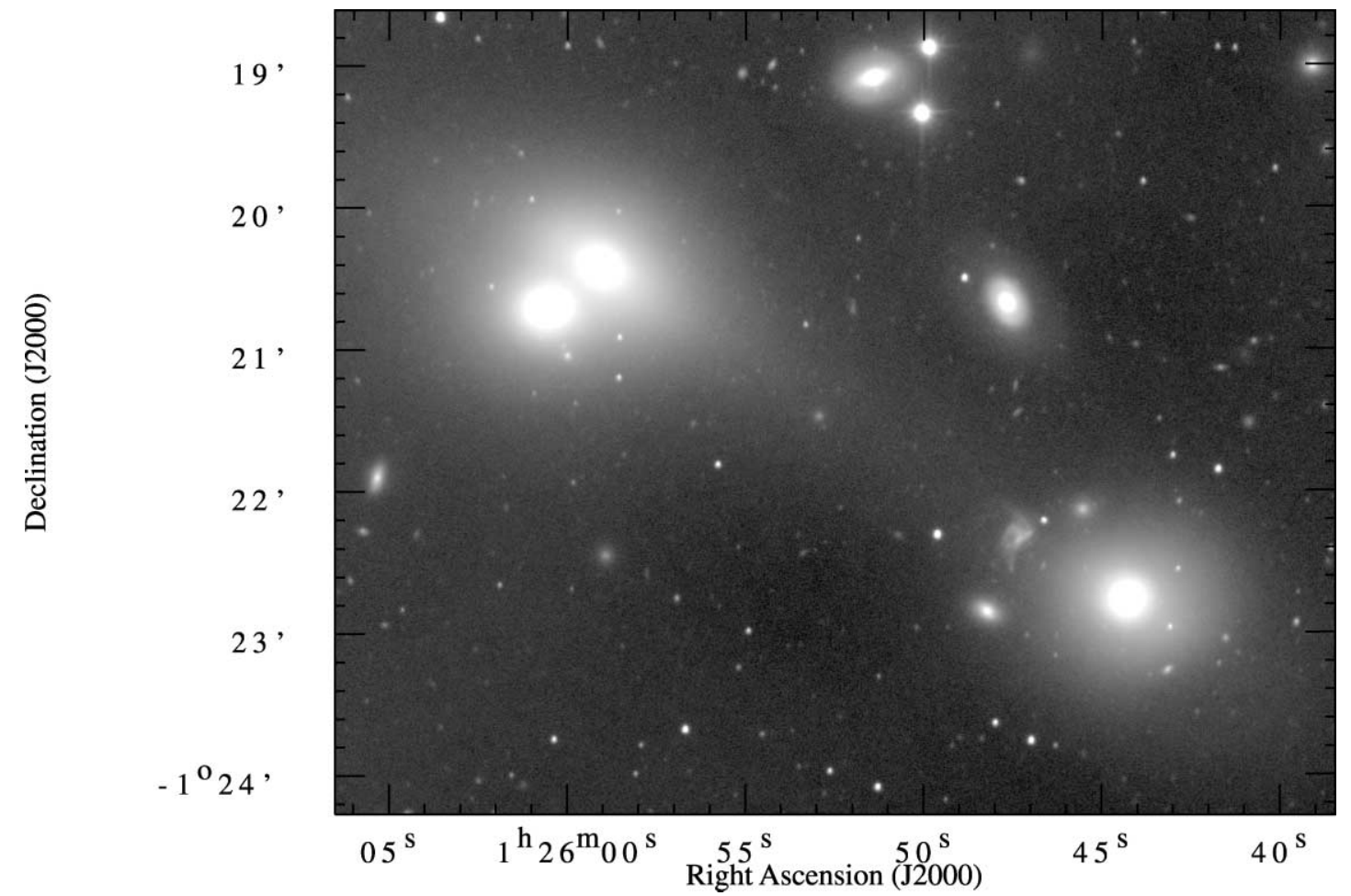

FIG. 4.-PFCam R-band co-add, cropped to highlight the faint bridge of stars that connects NGC 541 with the interacting pair NGC 545/547. MO lies in this bridge, close to NGC 541. 


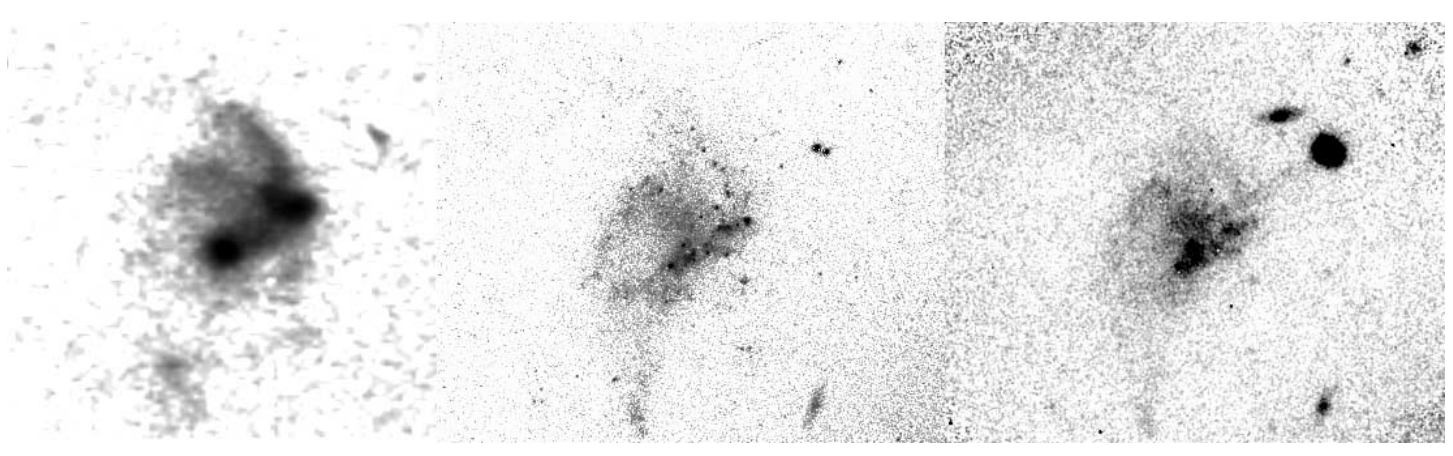

FIG. 5. - Left: Continuum-subtracted ATT H $\alpha$ emission image of MO. Center: HST F555W (stellar continuum) image. Right: Keck NIRC $K^{\prime}$ image. All three images are aligned and show the same $1.0 \operatorname{arcmin}^{2}$ region of sky.

presumably due to differences in the narrowband filter transmission curves (since the same $R$-band filter was used for both fields), and the fact that the 668 filter samples a different part of the SEDs of the objects in the field than the 658 filter. The latter should be relatively unimportant due to the relatively small shift in wavelength between the two filters, except in the case of strong emission lines that may fall in one filter, but not the other (e.g., $\mathrm{H} \alpha$ in particular). Although this is the case for the main targets in each field, the good fit obtained when all binned pixels are included suggests that this effect is negligible, and that the main difference is due to the relative transmission of the filters. Therefore, we scale the $\mathrm{H} \alpha$ fluxes measured from MO by the ratio of the slopes (i.e., the MO fluxes are multiplied by 0.791 ) to account for this. The resulting continuum-free $\mathrm{H} \alpha$ image is shown in Figure 5 and has a resolution of 1".3 FWHM.

The total flux integrated within an equivalent $16^{\prime \prime}$ diameter aperture (after correction for Galactic reddening; see $\S 3.1$ ) is determined to be $8.6 \times 10^{-14}$ ergs s${ }^{-1} \mathrm{~cm}^{-2}$, yielding an $\mathrm{H} \alpha$ luminosity of $6.6 \times 10^{40} \mathrm{ergs} \mathrm{s}^{-1}$. This is approximately twice the value obtained by van Breugel et al. (1985); some $14 \%$ of this increase is attributable to the different cosmology used in their paper, and the remainder is presumably due to improvements in both the sensitivity and calibration of the current $\mathrm{H} \alpha$ observations.

The $\mathrm{H} \alpha$ image is similar in its morphological features to the optical continuum images, with a strong concentration of $\mathrm{H}$ II regions and their associated clusters in the linear barlike feature facing the incoming jet, and more diffuse filaments of emission on the downstream side. These are more conspicuous in $\mathrm{H} \alpha$ than in the continuum, suggesting that the clusters in this region are somewhat younger than in the bar. MO-South is more clearly defined in $\mathrm{H} \alpha$. The overall morphology is highly suggestive of a jet-cloud interaction, with subsequent star formation triggered by the jet.

\subsection{Keck ESI R-Band and NIRC $J$ - and $K^{\prime}$-Band Imaging}

To complement the HST $V$ - and $I$-band observations, and to provide spectral energy distribution data over the widest possible range, we obtained Keck $R-, J_{-}$, and $K^{\prime}$-band images.

In the $R$ band, a $75 \mathrm{~s}$ image centered on MO was obtained with the Echellette Spectrograph and Imager (ESI; Sheinis et al. 2002) on Keck II on 2002 September 7, and calibrated and reduced in IRAF in the standard manner. The FWHM of the seeing was 0.7 , and the image is shown in Figure 6. This image clearly shows the existence of a fainter structure extending about $30^{\prime \prime}$ downstream of the bright bar of MO-North, which was also faintly detected in the $\mathrm{H} \alpha$ image.

In $J$ and $K^{\prime}, 600 \mathrm{~s}$ exposures were obtained with the Near Infrared Camera (NIRC) on Keck I on 2004 December 17. A five-position dither, with dither spacing $5^{\prime \prime}$ and $60 \mathrm{~s}$ per exposure $\left(3 \times 20 \mathrm{~s}\right.$ in $J, 4 \times 15 \mathrm{~s}$ in $\left.K^{\prime}\right)$, was used. Between each on-source dither position, a $40^{\prime \prime}$ nod to a nearby blank area of sky was made, and an additional $60 \mathrm{~s}$ exposure off source was made (yielding the same dither pattern as for the on-source exposures). The on-source images were registered and combined. The off-source images were combined with object masking and without registration to form a sky image, which was scaled and subtracted from the

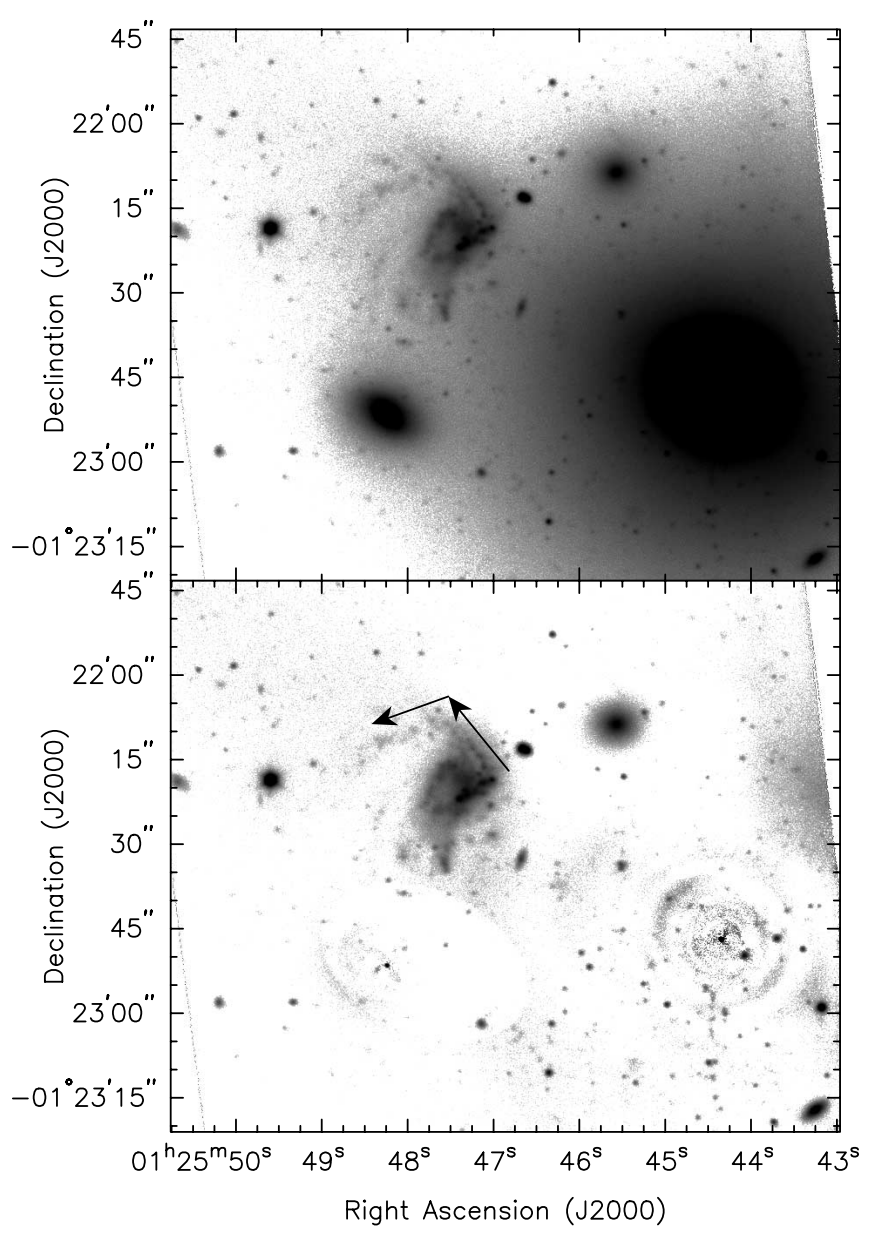

FIG. 6. - Keck ESI $R$-band image of MO (top), with elliptical galaxy models subtracted (bottom) as described in the text. Both images are displayed using the same logarithmic scaling. The arrows in the bottom panel illustrate the filamentary feature downstream of the central bar, discussed in $\S 2.5$. They are not intended to suggest that this feature necessarily formed due to flow of material from the central regions of MO. 

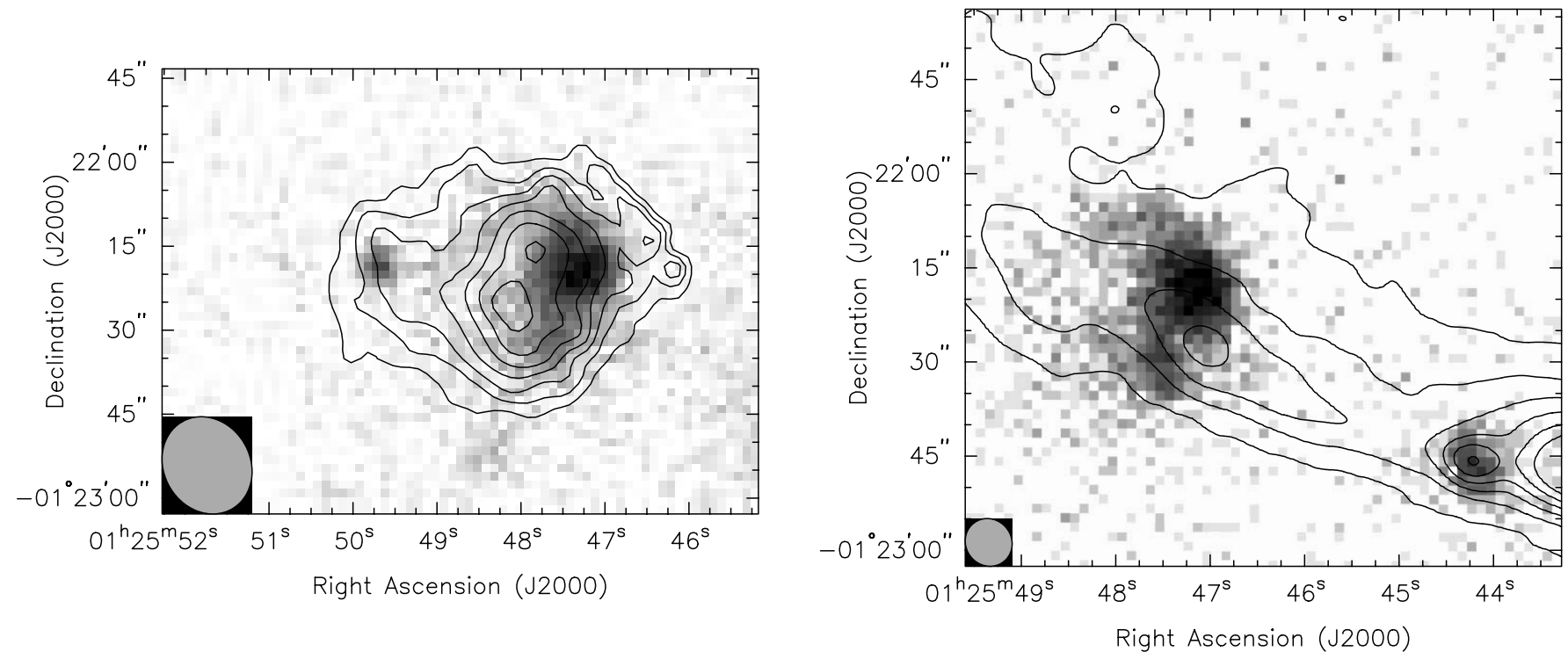

FIg. 7.-Left: GALEXNUV image, overlaid with H I contours from the VLA. The NUV image is shown with a logarithmic scaling to emphasize faint structures; note that the blob at $01^{\mathrm{h}} 25^{\mathrm{m}} 50^{\mathrm{s}}$ is a foreground star. $\mathrm{H}$ i contours are from $0.5-6.5 \mathrm{mJy}_{\text {beam }}^{-1} \mathrm{~km} \mathrm{~s}^{-1}$, at intervals of $1.0 \mathrm{mJy}$ beam ${ }^{-1} \mathrm{~km} \mathrm{~s}^{-1}$. The gray ellipse represents the $17^{\prime \prime} .9 \times 15^{\prime \prime} .4$ CLEAN beam $\left(\mathrm{PA}=30^{\circ}\right)$ of the $\mathrm{H}$ i observations. Right: GALEXFUV image (shown with a logarithmic scaling), overlaid with radio continuum contours from the VLA. Radio contours are from $2.0-17.0 \mathrm{mJy}_{\text {beam }}^{-1}$ at intervals of $3.0 \mathrm{mJy}_{\text {beam }}{ }^{-1}$. The gray ellipse represents the $7.8 \times 7^{\prime \prime} .3 \mathrm{CLEAN}$ beam $\left(\mathrm{PA}=36^{\circ}\right)$ of the radio observations. Note that the two panels are not shown to the same scale; the relationship between the radio jet and the $\mathrm{H}$ I is better seen in Fig. 9.

on-source image. The final reduced images cover a field of $100^{\prime \prime} \times$ $100^{\prime \prime}$ with a FWHM of 0."5.

The $K^{\prime}$-band image is shown as the third panel in Figure 5. The $K^{\prime}$-band morphology very closely resembles the optical F555W image taken with the $H S T$. This shows that there cannot be a large old stellar population present in $\mathrm{MO}$, and that the $K^{\prime}$-band light is probably dominated by young supergiants.

\subsection{GALEX Imaging}

The field containing the NGC 541/545/547 galaxies in Abell 194 was observed as part of the GALEX (Martin et al. 2005) Nearby Galaxy Survey (Gil de Paz et al. 2006) in the near-UV

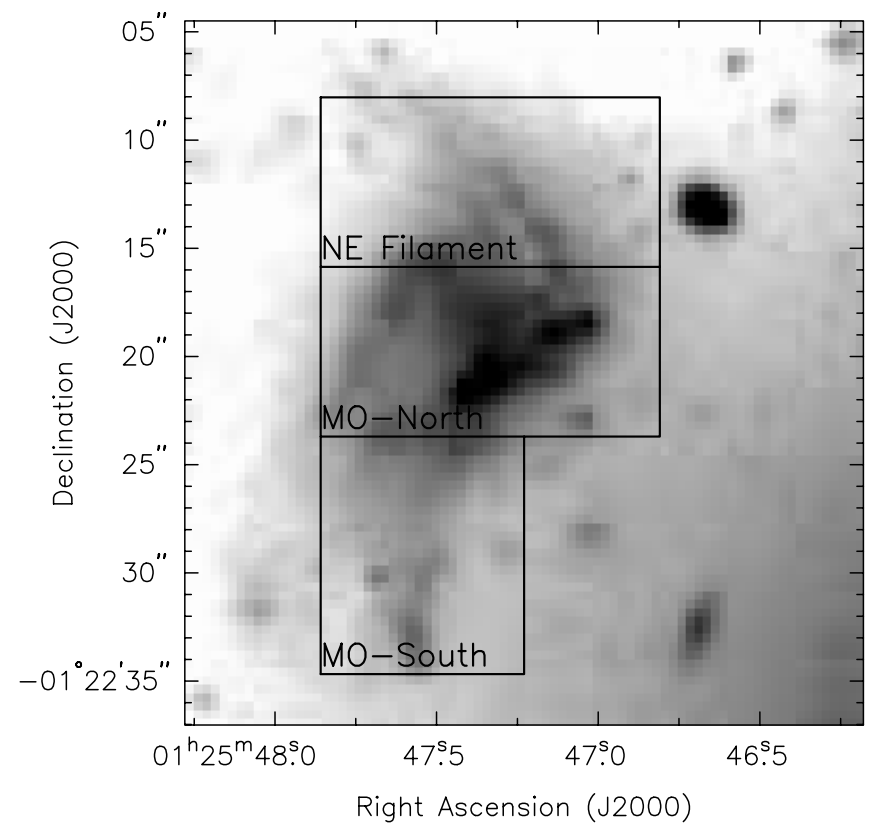

FIG. 8.-Binned ESI-R image of MO, overlaid with boxes showing the regions used for the three-aperture photometry (see text).
(NUV, 1750-2800 $\AA$ ) and far-UV (FUV, 1350-1750 §) passbands on 2003 October 23. Exposure times were $1700 \mathrm{~s}$ in each band. The data were reduced and photometrically calibrated using the GALEX pipeline (Morrissey et al. 2005). The $5 \sigma \mathrm{AB} \mathrm{lim}-$ iting magnitude in both bands is $m \sim 23$, corresponding to a surface brightness sensitivity of $\sim 26.5 \mathrm{mag} \operatorname{arcsec}^{-2}$ averaged over the PSF. The FWHM in the NUV and FUV images is 5".6 and 4.0 , respectively.

MO was prominently detected in both bands and shows similar overall structure to the optical, $\mathrm{H} \alpha$, and near-IR images, even given the relatively low resolution of GALEX. The GALEX field of view is large and covers the central 1.2 of the Abell 194 cluster, yet MO is among the brightest resolved UV structures in this entire field.

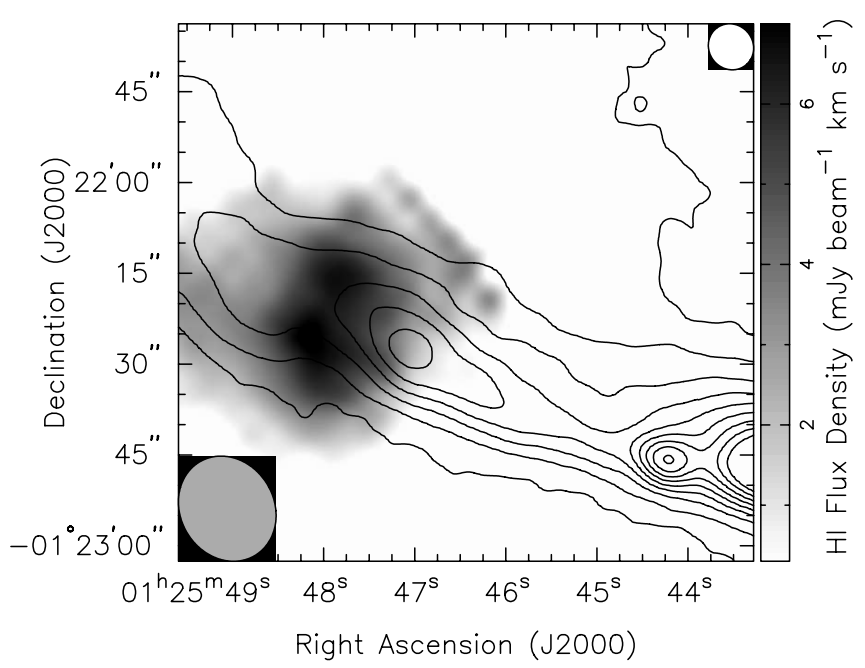

FIG. 9.- Map of the $\mathrm{H}$ I cloud adjoining MO, with radio continuum contours from the VLA overlaid. The $\mathrm{H}$ I cloud is shown with a linear scaling from 0.3$7 \mathrm{mJy}$ beam ${ }^{-1} \mathrm{~km} \mathrm{~s}^{-1}$, corresponding to the gray-scale wedge at right. Continuum contours are from $1-17 \mathrm{mJy}_{\text {beam }}^{-1}$ at $2 \mathrm{mJy}_{\text {beam }}{ }^{-1}$ intervals. The $\mathrm{H}$ I beam is shown as a gray ellipse (lower left), and the continuum beam is shown as a white ellipse (upper right). 


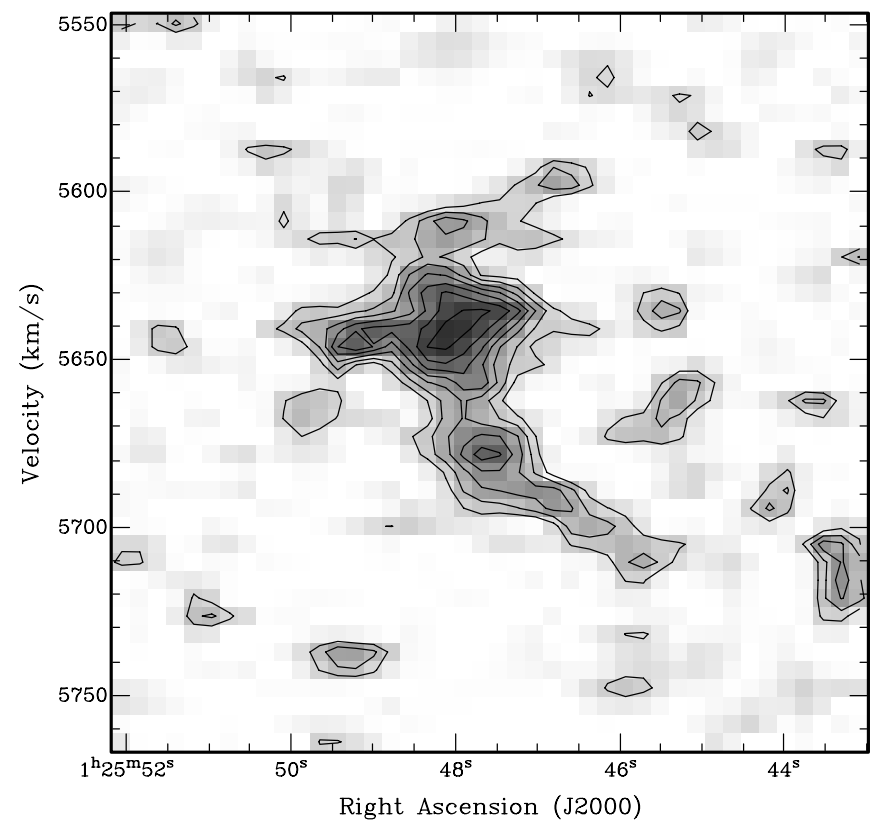

FIG. 10.-Velocity slice through the MO H I map, showing velocity shear near the outer boundaries of the $\mathrm{H}$ I cloud and jet. Contour levels are 0.5, 1.0, 1.5, 2.0, $2.5,3.0 \mathrm{mJy}$ beam $^{-1}$, and encode the same information as the gray-scale.

The images were overlaid with the VLA radio continuum and H I observations ( $\S 2.7$ ), as shown in Figure 7. In both UV bands, MO appears as a double source, with a clear separation of MONorth and MO-South straddling the central axis of the radio jet, with MO-North being the brightest component. Both components are clearly extended downstream, paralleling the radio jet, as in the optical and near-IR images.

The total observed near-UV flux from MO was determined to be $1.78 \times 10^{-15} \mathrm{ergs} \mathrm{s}^{-1} \mathrm{~cm}^{-2} \AA^{-1}$, and the far-UV flux was $3.63 \times 10^{-15} \mathrm{ergs} \mathrm{s}^{-1} \mathrm{~cm}^{-2} \AA^{-1}$, yielding values corrected for

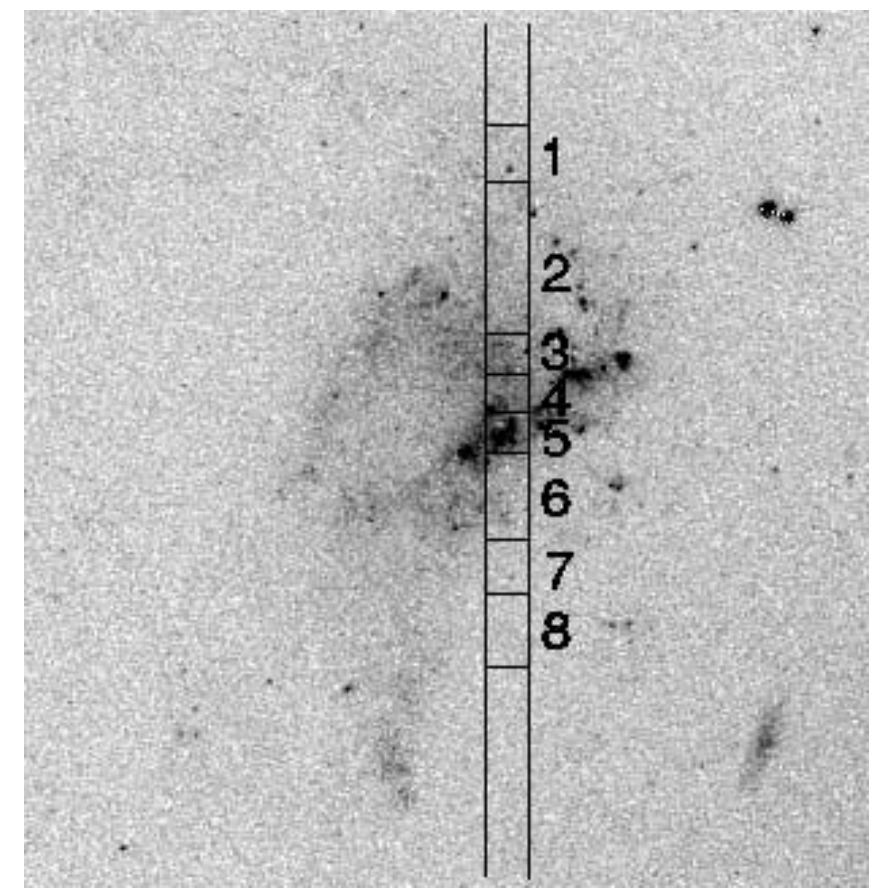

Galactic extinction of $2.57 \times 10^{-15} \mathrm{ergs} \mathrm{s}^{-1} \mathrm{~cm}^{-2} \AA^{-1}$ and $5.11 \times$ $10^{-15} \operatorname{ergs~s}^{-1} \mathrm{~cm}^{-2} \AA^{-1}$, respectively. This is in good agreement with a flux of $2.96 \times 10^{-15} \mathrm{ergs} \mathrm{s}^{-1} \mathrm{~cm}^{-2} \AA^{-1}$ at $1350 \AA$ from an archival International Ultraviolet Explorer spectrum (K. Chambers 2005, private communication). We also extracted photometry for three smaller, rectangular apertures, centered on MO-North, the northeast (NE) filament downstream from MONorth, and MO-South (Fig. 8). These GALEX photometry data allow us to put strict limits on the stellar age distribution of $\mathrm{MO}$, as we discuss in $\S 4.2$.

\subsection{The Radio H I VLA Observations}

To search for cold gas associated with MO and its surroundings, we performed $\mathrm{H}$ I observations with the VLA in its C-array configuration on 2002 April 2. The total integration time onsource was $4.3 \mathrm{hr}$. A $3 \mathrm{MHz}$ bandwidth was used, with $128 \mathrm{spec}-$ tral channels, covering the heliocentric velocity range 5305$5815 \mathrm{~km} \mathrm{~s}^{-1}$ (the full observing band is $600 \mathrm{~km} \mathrm{~s}^{-1}$, but on either side of this band are a few channels that are not usable). The data were calibrated following standard procedures using the MIRIAD software package. A spectral-line data cube was made using robust weighting (robustness $=0.5$ ) giving a spatial resolution of $18^{\prime \prime} \times 15^{\prime \prime}\left(\mathrm{PA}=29^{\circ}\right)$. Hanning smoothing was applied to the data cube, resulting in a velocity resolution of $10.3 \mathrm{~km} \mathrm{~s}^{-1}$. The noise in the final data cube is $0.54 \mathrm{mJy} \mathrm{beam}^{-1}$.

Within the entire primary beam $(0.5)$, the only $\mathrm{H}$ i that we detect is from $\mathrm{MO}$ (Fig. 9), but this may be due to our relatively narrow band, covering only $\sim 500 \mathrm{~km} \mathrm{~s}^{-1}$ (the velocity range of the cluster is surely larger than that). Indeed, a short test observation with the WSRT in 2004 August of MO with a wider band of $10 \mathrm{MHz}$ covering $2000 \mathrm{~km} \mathrm{~s}^{-1}$ (using 1024 channels) showed at least three other $\mathrm{H}$ i objects, apart from MO, at velocities just outside the band used with the VLA for the MO observations. A more detailed observation of Abell 194 with the WSRT would likely reveal more objects.

The H I emission is dominated by two "blobs" sitting on either side of the jet and is offset from MO, with the brightest $\mathrm{H}_{\mathrm{I}}$

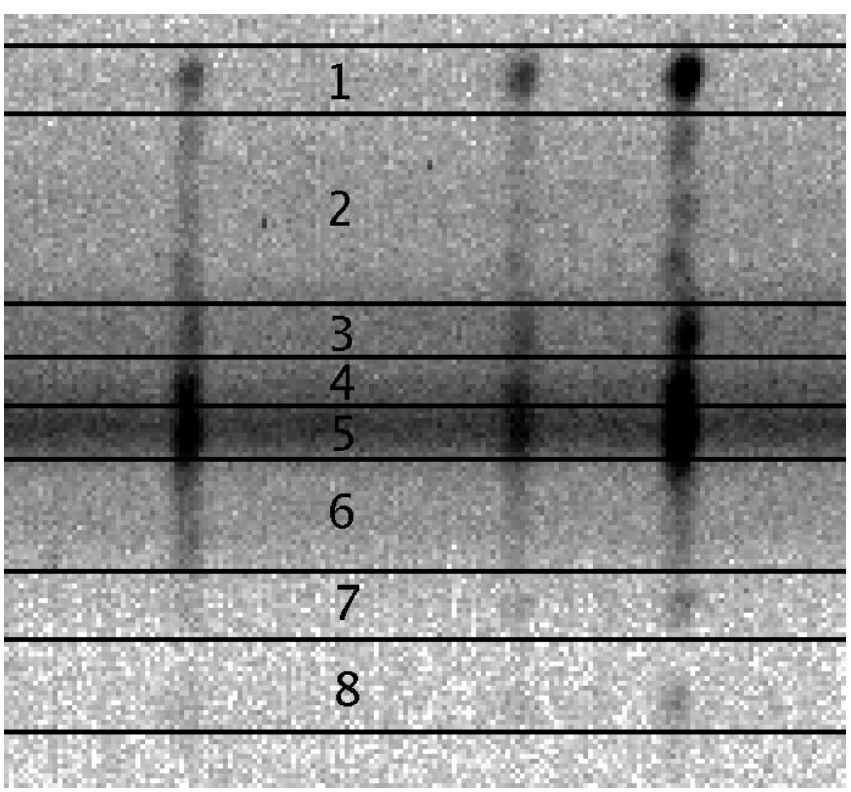

FIG. 11.-LRIS slit position (left), overlaid on the F555W image of MO (the width of the slit is accurately shown). Numbered subapertures are the same as shown on the

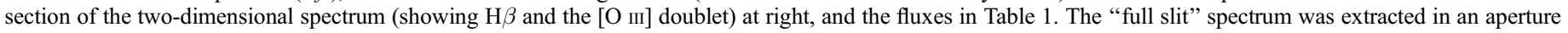
covering subapertures $1-8$. 


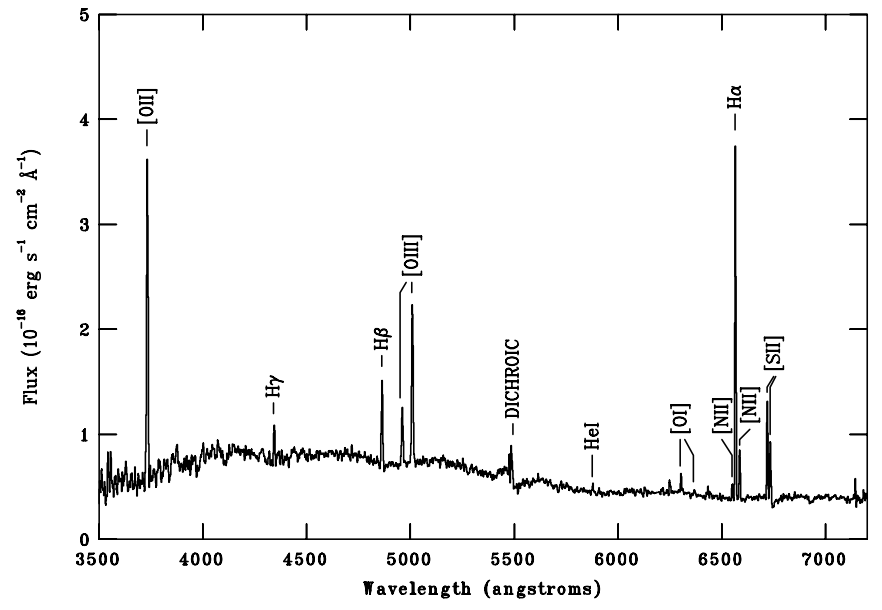

FIG. 12.-Combined blue and red side LRIS spectra (1800 s), smoothed with a 3 pixel boxcar filter.

$\sim 10^{\prime \prime}$ (3.6 kpc) downstream from the peak of the $\mathrm{H} \alpha$ emission (the main star formation region). The position of these maxima is coincident with the downstream extensions of MO-North and MO-South noted in the GALEX image. In addition, two faint $\mathrm{H}$ I filaments are found extended along the edges of the jet. One of these lies to the north of MO-North, upstream in the sense of the jet motion. The other forms a continuation of MO-South downstream. The jet itself changes direction and decollimates downstream from the $\mathrm{H}$ I clouds, which suggests that it is the jet-gas interaction that has disrupted the jet.

The H I kinematics, along an east-west slice (see Fig. 10) show that most of the $\mathrm{H}$ I gas is relatively quiescent, with a average velocity $v_{\mathrm{H}_{\mathrm{I}}} \sim 5640 \mathrm{~km} \mathrm{~s}^{-1}$, or $z=0.0188$. This is in excellent agreement with the redshift $z=0.0189$ of the star-forming region obtained from the LRIS spectroscopy. To make this plot, the data were integrated in declination over about $35^{\prime \prime}$. This was done to show the faint low- and high-velocity wings. There is a smooth north-south velocity gradient, and the North and South $\mathrm{H}$ I blobs have a velocity difference of about $\sim 50 \mathrm{~km} \mathrm{~s}^{-1}$.
TABLE 2

Absorption Lines from LRIS SPECTROSCOPY, Corrected for Galactic Reddening

\begin{tabular}{|c|c|c|c|}
\hline Line & $\begin{array}{r}\lambda_{\text {rest }} \\
(\AA)\end{array}$ & Flux $^{1}$ & $\begin{array}{c}\mathrm{EW}_{0} \\
(\AA)\end{array}$ \\
\hline 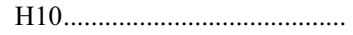 & 3798 & $218 \pm 2$ & $3 \pm 1$ \\
\hline Н9 & 3835 & $262 \pm 2$ & $3 \pm 1$ \\
\hline 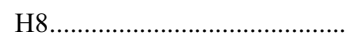 & 3889 & $223 \pm 2$ & $3 \pm 1$ \\
\hline $\mathrm{H} \delta$ & 4102 & $260 \pm 2$ & $3 \pm 1$ \\
\hline $\mathrm{H} \gamma$ & 4340 & $363 \pm 2$ & $4 \pm 1$ \\
\hline 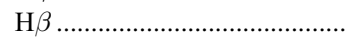 & 4861 & $247 \pm 1$ & $3 \pm 1$ \\
\hline
\end{tabular}

Note.-Line fluxes are given in $10^{-18} \mathrm{ergs} \mathrm{s}^{-1} \mathrm{~cm}^{-2}$.

We derive a total $\mathrm{H}$ I mass for MO of $4.9 \times 10^{8} M_{\odot}$. This was done assuming the $\mathrm{H}_{\mathrm{I}}$ is optically thin. Then the mass follows from $M_{\mathrm{H}_{\mathrm{I}}}=2.35 \times 10^{5} \mathrm{FD}^{2}$, where $F$ is the flux integral in $\mathrm{Jy} \mathrm{km} \mathrm{s}{ }^{-1}$ (i.e., the integral $\int S d v$, where $S$ is the detected flux in a channel and $v$ is velocity) and $D$ is the distance to MO in Mpc.

The mass of $\mathrm{H}$ I is approximately evenly divided between the two main blobs straddling the jet (Fig. 9). The H I column density through the center of each of the blobs is $n_{\mathrm{H}} \sim 4.3 \times 10^{20} \mathrm{~cm}^{-2}$ and $n_{\mathrm{H}} \sim 4.7 \times 10^{20} \mathrm{~cm}^{-2}$ for the northern and southern blobs, respectively. The total (projected) extent of the $\mathrm{H}$ I emission is approximately $275 \mathrm{kpc}^{2}$.

\subsection{Keck LRIS Spectroscopy}

Any potential spatial variations in the stellar populations or ages are best studied through spatially resolved spectroscopy. To investigate any spatial variations in ionization and anomalous kinematics across MO, we obtained a high signal-to-noise ratio (S/N) long-slit spectrum using the Low Resolution Imaging Spectrometer (LRIS; Oke et al. 1995) on Keck I on 2004 January 20. The D560 dichroic was used with a slit width of 1".5. The total exposure time was $1800 \mathrm{~s}$. On the blue side, a 300 line $\mathrm{mm}^{-1}$ grism blazed at $5000 \AA$ was employed, giving $1.43 \AA$ pixel $^{-1}$ and spectral resolution $10.7 \AA$. On the red side, a 400 line $\mathrm{mm}^{-1}$ grating blazed at $8500 \AA$ was used, giving $1.86 \AA$ pixel $^{-1}$ and spectral

TABLE 1

Equivalent Widths and Galactic-Reddening-Corrected Line Fluxes from LRIS Spectroscopy

\begin{tabular}{|c|c|c|c|c|c|c|c|c|c|c|c|}
\hline \multirow[b]{2}{*}{ LINE } & \multirow{2}{*}{$\begin{array}{r}\lambda_{\text {rest }} \\
(\AA)\end{array}$} & \multirow{2}{*}{$\frac{\mathrm{EW}_{0}(\AA)}{\text { Full Slit }}$} & \multicolumn{9}{|c|}{ FLuX $(\mathrm{H} \beta=100)$} \\
\hline & & & Full Slit & Ap 1 & Ap 2 & Ap 3 & Ap 4 & Ap 5 & Ap 6 & Ap 7 & Ap 8 \\
\hline$[\mathrm{O}$ II $] \ldots \ldots \ldots \ldots \ldots \ldots \ldots \ldots \ldots \ldots \ldots \ldots$ & 3727 & $63.6 \pm 0.4$ & 359.0 & 221 & 406 & 449 & 305 & 454 & 362 & 289 & 240 \\
\hline$[\mathrm{Ne}$ III $] \ldots \ldots \ldots \ldots \ldots \ldots \ldots$ & 3869 & $\ldots$ & $\ldots$ & $\ldots$ & $\ldots$ & 40 & 28 & $\ldots$ & 31 & $\ldots$ & $\ldots$ \\
\hline 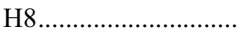 & 3889 & $1.0 \pm 0.3$ & 7.9 & $\ldots$ & $\ldots$ & $\ldots$ & $\ldots$ & $\ldots$ & $\ldots$ & $\ldots$ & $\ldots$ \\
\hline $\mathrm{H} \delta \ldots \ldots \ldots \ldots \ldots \ldots \ldots \ldots \ldots \ldots \ldots \ldots \ldots \ldots$ & 4102 & $1.1 \pm 0.3$ & 9.7 & $\ldots$ & $\ldots$ & $\ldots$ & $\ldots$ & $\ldots$ & $\ldots$ & $\ldots$ & $\ldots$ \\
\hline 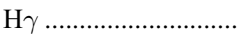 & 4340 & $4.7 \pm 0.3$ & 40.8 & 51 & 31 & 33 & 30 & 31 & 30 & $\ldots$ & $\ldots$ \\
\hline 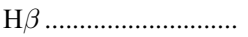 & 4861 & $12.4 \pm 0.3$ & 100.0 & 100 & 100 & 100 & 100 & 100 & 100 & 100 & 100 \\
\hline$[\mathrm{O}$ III $] \ldots \ldots \ldots \ldots \ldots \ldots \ldots$ & 4959 & $6.7 \pm 0.3$ & 60.7 & 115 & 115 & 59 & 85 & 49 & 36 & 67 & 87 \\
\hline$[\mathrm{O}$ III $] \ldots \ldots \ldots \ldots \ldots \ldots \ldots$ & 5007 & $19.4 \pm 0.3$ & 172.4 & 333 & 333 & 180 & 252 & 169 & 114 & 211 & 200 \\
\hline 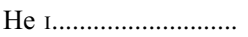 & 5876 & $1.7 \pm 0.4$ & 9.4 & $\ldots$ & 11 & 17 & 16 & 11 & 7 & $\ldots$ & $\ldots$ \\
\hline$[\mathrm{O}$ I $] \ldots \ldots \ldots \ldots \ldots \ldots \ldots \ldots \ldots \ldots \ldots \ldots \ldots$ & 6300 & $2.5 \pm 0.3$ & 14.1 & $\ldots$ & 22 & 28 & 20 & 11 & 16 & $\ldots$ & 46 \\
\hline 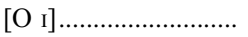 & 6363 & $1.0 \pm 0.3$ & 5.2 & $\ldots$ & 7 & $\ldots$ & 7 & $\ldots$ & $\ldots$ & $\ldots$ & $\ldots$ \\
\hline$\left[\mathrm{N}_{\text {III }}\right] \ldots \ldots \ldots \ldots \ldots \ldots \ldots \ldots \ldots \ldots \ldots \ldots$ & 6548 & $2.9 \pm 0.3$ & 13.6 & $\ldots$ & 15 & 7 & 23 & 16 & 10 & $\ldots$ & $\ldots$ \\
\hline 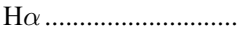 & 6563 & $58.6 \pm 0.3$ & 278.7 & 226 & 306 & 335 & 396 & 318 & 238 & 350 & 390 \\
\hline$\left[\mathrm{N}_{\text {II }}\right] \ldots \ldots \ldots \ldots \ldots \ldots \ldots \ldots \ldots \ldots \ldots \ldots$ & 6583 & $8.3 \pm 0.3$ & 39.5 & 14 & 44 & 54 & 59 & 46 & 36 & $\ldots$ & $\ldots$ \\
\hline$[\mathrm{S}$ II] & 6716 & $17.1 \pm 0.3$ & 78.7 & 31 & 94 & 105 & 114 & 81 & 74 & 100 & 120 \\
\hline$[\mathrm{S}$ пा] & 6731 & $12.2 \pm 0.3$ & 55.0 & 18 & 55 & 75 & 73 & 57 & 53 & 50 & $\ldots$ \\
\hline$F_{\mathrm{H} \beta}^{1} \cdots \cdots \cdots \cdots \cdots \cdots \cdots \cdots \cdots \cdots \cdots \cdots$ & $\ldots$ & $\ldots$ & $789 \pm 2$ & $84 \pm 3$ & $124 \pm 3$ & $57 \pm 3$ & $116 \pm 3$ & $140 \pm 3$ & $134 \pm 3$ & $18 \pm 3$ & $15 \pm 4$ \\
\hline
\end{tabular}

Notes.-Ap1-8 are subapertures of observation. $\mathrm{H} \beta$ line fluxes are given in $10^{-18} \mathrm{ergs} \mathrm{s}^{-1} \mathrm{~cm}^{-2}$. 
TABLE 3

Derived Quantities from the Observed Emission Lines from the LRIS Subapertures

\begin{tabular}{|c|c|c|c|c|c|c|c|c|}
\hline Quantity & Ap 1 & Ap 2 & Ap 3 & Ap 4 & Ap 5 & Ap 6 & Ap 7 & Ap 8 \\
\hline$E(B-V)$ & -0.10 & 0.03 & 0.06 & 0.13 & 0.04 & -0.08 & 0.08 & 0.13 \\
\hline Subaperture width $(\operatorname{arcsec})$ & 1.89 & 5.13 & 1.35 & 1.22 & 1.35 & 2.97 & 1.78 & 2.43 \\
\hline Subaperture size $\left(\mathrm{kpc}^{2}\right) \ldots \ldots \ldots \ldots \ldots \ldots \ldots$ & 0.397 & 1.076 & 0.283 & 0.256 & 0.283 & 0.623 & 0.373 & 0.510 \\
\hline $\operatorname{SFR}(\mathrm{H} \alpha) 10^{-3} M_{\odot} \mathrm{yr}^{-1} \mathrm{kpc}^{-2} \ldots \ldots \ldots$ & 2.90 & 2.14 & 4.09 & 10.90 & 9.56 & 3.12 & 1.02 & 0.70 \\
\hline 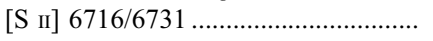 & 1.73 & 1.71 & 1.40 & 1.56 & 1.43 & 1.39 & 2.00 & $\cdots$ \\
\hline $\log \mathcal{U}^{\mathrm{a}}$ & -2.6 & -3.0 & -3.2 & -3.0 & -3.0 & -3.0 & $\ldots$ & $\ldots$ \\
\hline
\end{tabular}

${ }^{\text {a }}$ Determined from the $[\mathrm{N}$ II $] / \mathrm{H} \alpha$ diagnostic (Kewley \& Dopita 2002), assuming constant metallicity of $Z=0.5 Z_{\odot}$.

resolution $6.8 \AA$. The data were reduced in IRAF, with cosmic ray removal, sky line subtraction, wavelength calibration, and flux calibration performed in the standard manner. The blueand red-side spectra were combined using SCOMBINE in IRAF, and the resultant pixel scale was $1.41 \AA \mathrm{pixel}^{-1}$, so the resulting spectra were smoothed with a 3 pixel boxcar filter to improve the $\mathrm{S} / \mathrm{N}$. Spectra were extracted using several apertures and were corrected for Galactic extinction using $E(B-V)=0.045$ and $R_{V}=3.1$ (Schlegel et al. 1998).

A large, $21^{\prime \prime}$-long aperture spectrum (just covering subapertures $1-8$ as shown in Fig. 11) was extracted to obtain an integrated spectrum encompassing all of the emission from $\mathrm{MO}$ along the 1".5-wide slit. The extracted spectrum is shown in Figure 12. From this, the redshift of MO was determined to be $z=0.0189$ (similar to the value found by van Breugel et al. 1985). The spectra were Doppler corrected to zero redshift. The measured emissionline properties integrated along the slit, and for various subapertures described below, are presented in Table 1 .

Fluxes were measured from Gaussian fits to the lines, with $1 \sigma$ errors estimated from Monte Carlo noise simulations (noise estimates and gain were determined from measurements of the spectrum).

The strengths of the Balmer absorption lines (Table 2) were measured by fitting a Gaussian to the wings of the absorption underlying the Balmer emission components. Quoted fluxes are the amount of absorbed flux based on a fit to the continuum after subtraction of a Gaussian model for the emission component. The emission-line fluxes in Table 1 are corrected for underlying absorption in the line cores.

In addition, spectra were extracted in eight subapertures, chosen to coincide with regions of the two-dimensional spectrum and images that appeared to have relatively homogeneous properties across them (see Fig. 11). The properties of the measured emission lines are also shown in Table 1 . There was insufficient $\mathrm{S} / \mathrm{N}$ in the absorption lines in the subapertures to accurately determine absorption line strengths at these positions.

Line ratios, which depend on the measurement of lines on both the red and blue sides, are less reliable for the subapertures than for the integrated spectrophotometry, due to the difficulty of measuring exactly the same region of the two-dimensional spectrum in apertures of only a few pixels length. This is presumably part of the source of the variation in the estimated $E(B-V)$ seen in Table 3. Line ratios determined using lines from only the red or blue sides are unaffected by this additional source of uncertainty. Since the slit is oriented north-south, the observations will to some extent be affected by atmospheric differential refraction (Filippenko 1982). This was minimized by observing at relatively low air mass $(Z<1.20)$, but dispersion still has the effect of offsetting the physical regions of the source where lines at the red and blue ends of the spectrum are measured (as badly as 0.9

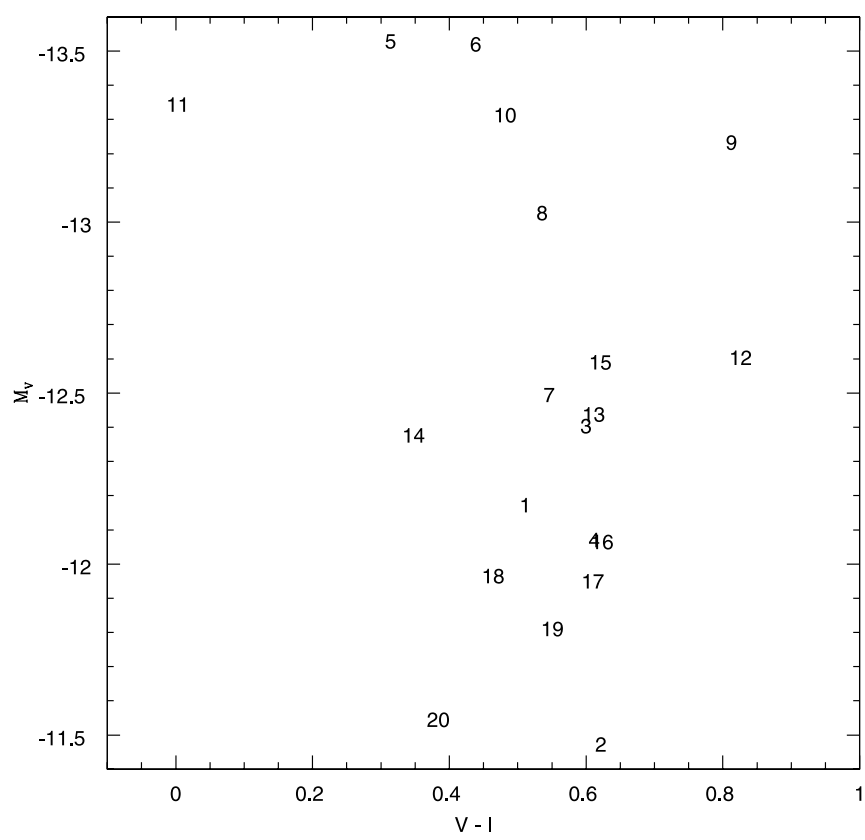

FIG. 13.-Left: F555W image of MO, showing the apertures used for F555W/ F814W photometry of "blobs," numbered sequentially from south to north. Right: Photometry of the blobs in MO, converted from F555W/F814W to Johnson $V$ and $I$, and to absolute magnitudes in $V$. Colors and magnitudes are typical for young star clusters. 
in the case of [O II] and [S II]). In the case of ratios such as $\mathrm{H} \alpha / \mathrm{H} \beta$, the differential refraction is at worst around 0.36 , i.e., around $25 \%$ of the width of the slit. Inspection of Figure 13 suggests that the extent of the extended emission compared to the slit width is such that a shift of this magnitude ought not to produce wild fluctuations in line ratios, especially since the seeing at the time of the LRIS observations $\left(0^{\prime \prime} 84\right.$, some 5 times worse than that of the F555W image in Fig. 13), will tend to wash out features at smaller scales.

In an extreme case where the flux of $\mathrm{H} \alpha$ or $\mathrm{H} \beta$ was different by a factor of 2 in the $25 \%$ of the slit discussed above, this could lead to errors in the computed extinction values of $E(B-V)_{\mathrm{s}} \sim 0.08$ (see $\S 3.1)$. The fact that the calculated value of $E(B-V)_{s}$ for the whole slit is so close to zero [formally, $E(B-V)_{s}=-0.01$ ] rather than, for example, strongly negative, suggests that these assertions are reasonable. This effect is presumably somewhat more important on smaller scales (e.g., the individual slitlets), and so may be an additional source of uncertainty (along with the difficulty of measuring the same region on the red and blue sides of the spectrum, discussed above) in the $E(B-V)$ values in Table 3. Since in practice, for the whole slit, we do not expect variations in flux of a factor of 2 on scales smaller than the atmospheric seeing, we assume that atmospheric dispersion is not a major source of error in our analysis.

The astute reader will note that the total $\mathrm{H} \beta$ flux quoted for the "full slit" in Table 1 is around $15 \%$ larger than the sum of the fluxes from the individual subapertures. This is again due to the difficulty of placing individual subapertures to subpixel precision, but as noted above does not affect ratios as measured in individual apertures.

The line ratios observed in these spectra confirm that the line emission is due to $\mathrm{H}$ II regions throughout MO (Veilleux \& Osterbrock 1987; Dopita et al. 2000). In particular, there is no evidence of shock-excited gas, which would reveal itself by very large $[\mathrm{N} \mathrm{II}] / \mathrm{H} \beta$ or $[\mathrm{O}$ II] $/ \mathrm{H} \beta$ ratios (Dopita et al. 1984). Nor does the $[\mathrm{O}$ I] $/ \mathrm{H} \alpha$ ratio become sufficiently large to put it in the shockexcited classification (Dopita \& Sutherland 1995). Furthermore, the kinematics show that the ionized gas is quiescent. There is no evidence for large velocity gradients, at least across the jet in the north-south slit direction. However, since shock-excited gas is more likely to reveal itself at low flux levels, it might be of interest to obtain a high-S/N spectroscopy along the jet direction.

\section{DERIVED QUANTITIES}

\subsection{Reddening}

The measured Balmer line ratio $\mathrm{H} \alpha / \mathrm{H} \beta$ from the full LRIS spectrum (after correction for Galactic reddening) was found to be $2.790 \pm 0.008$. Following Calzetti et al. (1994) and Calzetti (2001), this is consistent with an intrinsic (continuum) reddening of $E(B-V)_{s} \sim 0$.

We also calculated values of $E(B-V)_{s}$ for the subapertures (see Table 3). These show some variation, including values as negative as -0.10 . We believe these determinations to be less reliable than the full spectrum value for reasons discussed in $\S 2.8$; errors introduced by atmospheric dispersion and by not measuring the same part of the spectrum in the red and blue sides can probably account for any deviations from $E(B-V)_{s}=0$ in the subapertures.

We likewise used the (Galactic reddening corrected) broadband UV fluxes from GALEX to calculate $\beta$, the UV continuum slope (Calzetti et al. 1994), and obtained a value of $\beta=-1.734$, again yielding $E(B-V)_{s} \sim 0$, in excellent agreement with the line ratio results.
These more accurate measurements imply that there is essentially no dust obscuration along the line of sight to the emissionline regions of MO, and supersede those of van Breugel et al. (1985) who obtained $E(B-V)=0.27$. It is possible, however, that some of the variation in calculated values of $E(B-V)$ in the subapertures is in fact due to the presence of localized dust. If the dust is mixed in with the emission-line gas and stars to some extent (rather than a foreground screen, which is the assumption in the extinction calculations), then dust mass can be somewhat higher for a given $E(B-V)$ than otherwise.

\subsection{Metallicity}

As remarked above, the emission-line ratios are typical for those of $\mathrm{H}$ II regions, showing that the emission is dominated by star-forming regions, and so confirming the earlier conclusions by van Breugel et al. (1985). The high quality of our data allows us to obtain additional, more accurate diagnostics.

We determined the metallicity of MO using the method outlined in Kewley \& Dopita (2002). This yields a value of $\log (\mathrm{O} / \mathrm{H})+$ $12=8.6 \pm 0.1$, equivalent to a metallicity $Z \sim 0.5 Z_{\odot}$. The mean ionization parameter was determined to be $q \approx 3 \times 10^{7} \mathrm{~cm} \mathrm{~s}^{-1}$ $\left(\mathcal{U}=10^{-3}\right.$; see eq. [2]). This is within the normal values expected for extragalactic H II regions (Dopita et al. 2000).

\subsection{Star Formation Rates}

Applying the relation between $\mathrm{H} \alpha$ luminosity and star formation rate (SFR; Dopita \& Ryder 1994; Kennicutt 1998; Panuzzo et al. 2003),

$$
\left(\frac{\mathrm{SFR}_{\mathrm{H} \alpha}}{M_{\odot} \mathrm{yr}^{-1}}\right)=(7.0-7.9) \times 10^{-42}\left(\frac{L_{\mathrm{H} \alpha}}{\operatorname{ergs~s}^{-1}}\right),
$$

we used the measured $\mathrm{H} \alpha$ flux from the ATT images to obtain a total SFR within $\mathrm{MO}$ of $\sim 0.5 M_{\odot} \mathrm{yr}^{-1}$. This estimate is sensitive to the form of the initial mass function assumed for high-mass stars, and therefore has an uncertainty of order $30 \%$.

The local star formation rate increases, as expected, in the subapertures near the central blobs, where it is up to 1 order of magnitude higher than in the subapertures in the outer regions of MO. The measured star formation rates are given in Table 3, assuming the globally determined result of no intrinsic dust.

\subsection{Electron Densities}

The density-sensitive [S II] $\lambda 6716 / \lambda 6731$ doublet ratio was measured to be $1.431 \pm 0.01$ in the full spectrum. No other electron density diagnostics could be determined from our LRIS spectra, due to insufficient spectroscopic resolution and/or sensitivity. Using the five-level atom model of de Robertis et al. (1987), and assuming an electron temperature, $T_{e}=10^{4} \mathrm{~K}$, this yields an electron density of order $1-10 \mathrm{~cm}^{-3}$, averaged along the slit. However, for electron densities lower than approximately $100 \mathrm{~cm}^{-3}$, corresponding to values of this ratio $\gtrsim 1.32$, this line ratio becomes rather insensitive to electron density, and our measured electron density is therefore highly uncertain. Measurements of the ratio in the individual subapertures yield values ranging from $1.39-2.00$ (Table 3). Given that the errors on the ratios are rather high, due to a low $\mathrm{S} / \mathrm{N}$ in some subapertures, and that the $\mathrm{S}$ II lines are almost coincident with strong telluric lines whose subtraction introduces additional uncertainties, all we can conclude with certainty is that the electron densities are less than $100 \mathrm{~cm}^{-3}$.

\subsection{Ages of the Clusters}

As González Delgado et al. (1999) have shown, the strengths of the $\mathrm{He}_{\mathrm{I}}$ lines relative to $\mathrm{H} \beta$ are sensitive to the age of an $\mathrm{H}$ II 
region. We created our own photoionization models (appropriate to the metallicity of MO determined above) using MAPPINGS IIIr (Sutherland \& Dopita 1993; Dopita et al. 2002) in combination with Starburst99 (Leitherer et al. 1999) stellar-synthesis instantaneous-burst models, with a Salpeter IMF with lower mass limit of $1 M_{\odot}$ and upper mass limit of $100 M_{\odot}$. The use of a Salpeter IMF is appropriate for the modeling of both the ionizing flux and stellar continuum for a young $\left(<10^{8} \mathrm{yr}\right)$, recent starburst. The neglect of the $<1 M_{\odot}$ stars makes no difference to the results, as they contribute negligible luminosity at these early times. We find that the $\mathrm{He}$ I $\lambda 5876 / \mathrm{H} \beta$ line ratio remains near $0.1-0.12$ until $4 \mathrm{Myr}$, after which it declines steeply, becoming $<0.05$ after 5 Myr. The measured line ratios in Table 1 are therefore consistent with $\mathrm{H}$ II region ages of $<5 \mathrm{Myr}$, for all subapertures.

The $[\mathrm{O}$ III $] \lambda 5007 / \mathrm{H} \beta$ ratio is even more sensitive to age. In our models, it starts at around 4.5 , declines to $\sim 1.2$ by $2.5 \mathrm{Myr}$, to 0.5 by $3.0 \mathrm{Myr}$, and is negligible thereafter. We can therefore conclude that all the observed $\mathrm{H}$ II regions are younger than $\sim 3 \mathrm{Myr}$.

Comparison of the equivalent widths (EWs) of the Balmer nebular emission lines (Table 1) with Figure 16 of González Delgado et al. (1999) rules out continuous starburst models. The measured $\mathrm{H} \beta$ and $\mathrm{H} 8$ EWs both yield an instantaneous burst age of $6 \mathrm{Myr}$, and $\mathrm{H} \delta$ yields an instantaneous burst age of $8 \mathrm{Myr}$. Comparison of the measured EWs with Figures 3 and 4 of González Delgado et al. (1999) shows that ages greater than around $10 \mathrm{Myr}$ are excluded.

The discrepancies between the ages obtained from [O III] $\lambda 5007 / \mathrm{H} \beta$ and those from the EWs (discussed above) and SED fitting ( $\S 3.8$ ) may be due to a combination of some ongoing star formation, or possibly some contribution to the [O III] luminosity from shocked gas. Spectra with higher spatial resolution and $\mathrm{S} / \mathrm{N}$ might help resolve this.

\subsection{Number of Ionizing Photons}

Taking the Galactic reddening corrected ratio $\mathrm{H} \alpha / \mathrm{H} \beta=2.79$ determined from the full-aperture spectroscopy, and the $\mathrm{H} \alpha$ flux for the whole object from the imaging, we derive an $\mathrm{H} \beta$ luminosity of $2.37 \times 10^{40} \mathrm{ergs} \mathrm{s}^{-1}$. Comparing to González Delgado et al. (1999, Table 7), and assuming an age of $6 \mathrm{Myr}$ (from the emission-line EWs), we obtain a stellar mass of $1.9 \times 10^{7} M_{\odot}$, and $\log Q_{*}$ (the number of Lyman continuum photons) of 52.7. If we were instead to use the age of $3 \mathrm{Myr}$ obtained from the $\mathrm{H}$ II region spectroscopy, this would yield a stellar mass of $1.5 \times 10^{6} M_{\odot}$, so this determination is quite sensitive to age. The value of $\log Q_{*}$ depends only on the $\mathrm{H} \beta$ luminosity, and so is independent of age.

From Stasinska \& Leitherer (1996),

$$
\mathcal{U}=2.8 \times 10^{-20}\left(10^{4} / T_{e}\right)^{2 / 3}\left(Q_{*} n \epsilon^{2}\right)^{1 / 3}
$$

and with the measured values $\mathcal{U}=0.001, n=10 \mathrm{~cm}^{-3}, \log Q=$ 52.7 , and assuming $T_{e}=10^{4} \mathrm{~K}$, we obtain a nebular gas filling factor $\epsilon \sim 0.01$.

\subsection{Photometric Properties}

The NUV, FUV, ESI-R, F814W, $J$, and $K^{\prime}$ images were registered to the F555W image using the individual astrometric solutions. The F555W, F814W, $J$, and $K^{\prime}$ images were convolved to match the 0.72 seeing of the ESI-R image. We refrained from convolving all images to match the 5".6 FWHM of the NUV image, since this would result in such a significant loss of spatial resolution for the majority of the images. Photometry was performed in an $8^{\prime \prime}$ radius circular aperture to enclose all the emission from MO. Since MO is resolved in the GALEX images ( $\S 2.6$ ), we also obtained photometry for three subapertures (Fig. 8) centered on MO-North, the NE filament, and MO-South ( $\$ 4.2)$.

The elliptical galaxies in the ESI image (Fig. 6) were modeled using the tasks ELLIPSE and BMODEL in IRAF, and the best fitting models were subtracted from the original image. NGC 541 is difficult to fit in this image because it extends beyond the boundary of the frame to the right, but we were able to do a reasonable job of subtraction by carefully choosing and refining the fit parameters (see Fig. 6).

After subtraction of the elliptical galaxies, the bridge of stars joining NGC 541 and NGC 545/547 (see also Fig. 4) can be more clearly seen and more accurately measured. The bridge is around $24.5 \mathrm{mag} \operatorname{arcsec}^{-1}$, and extends over around $115^{\prime \prime} \times 45^{\prime \prime}$ $\left(\sim 700 \mathrm{kpc}^{2}\right)$; its total $R$-band luminosity is around $3 \times 10^{9} L_{\odot}$. From Nikogossyan et al. (1999), the gas mass within a $0.8 \mathrm{Mpc}$ radius of the center of the cluster is $1 \times 10^{13} M_{\odot}$, so we expect (by simple scaling, in the lack of more detailed information about the gas distribution) the gas mass in the bridge to be approximately $3 \times 10^{9} M_{\odot}$.

The bridge is approximately $18.7 \mathrm{mag}$ in the aperture used for the broadband photometry (i.e., approximately $15 \%$ as bright as $\mathrm{MO}$ ), and the portion of the NGC 541 model enclosed by this aperture is $55_{-20}^{+23} \%$ as bright as MO. Hence about $40 \%$ of the light in the aperture comes from NGC 541 and the bridge. Since a circular aperture was used, this includes parts of the aperture where MO is relatively faint, and parts where it is relatively bright, and so cannot be construed to be typical for the spectroscopic apertures or the individual pixels of the image. However, we consider the contribution from the bridge and elliptical when performing spectral synthesis modeling.

\subsection{Spectral Energy Distributions}

We computed SEDs using the seven bands of integrated $\left(8^{\prime \prime}\right.$ radius aperture) Galactic reddening corrected photometry for MO, converting magnitudes to fluxes in $\operatorname{ergs~s}^{-1} \mathrm{~cm}^{-2} \AA^{-1}$ using published zero points, and normalizing the fluxes to the flux measured in the F555W filter (approximately $V$-band) to facilitate model comparisons. A set of model spectra were created using Starburst99, with parameters as described in $\S 3.5$. These models were redshifted to match the redshift of MO, and convolved with the filter response functions, resulting in a set offluxes for each model. These were normalized to the F555W flux, and the best match between these fluxes and those from the observed photometry were determined by a $\chi^{2}$ minimization procedure.

In Figure 14, we plot $\chi_{\nu}^{2}=\chi^{2} / \nu$, where $\nu=6$ is the number of degrees of freedom (since seven filters are used, and the normalization takes away 1 degree of freedom). The best fitting (single-age) model was a $7.5 \mathrm{Myr}$ old SED with $\chi_{\nu}^{2}=0.22$. Models older than 14 Myr fit the photometry at $V$ through $K^{\prime}$ quite well, resulting in a broad minimum in the $\chi^{2}$ fit $\left(2 \lesssim \chi_{\nu}^{2} \lesssim 4\right)$. However, these older models do a poor job of reproducing the UV flux, and hence have higher $\chi^{2}$ than the 7.5 Myr model, which results in a sharp minimum in $\chi^{2}$, and reproduces the UV fluxes much better. A $\Delta \chi^{2}$ analysis, following Avni (1976) (with the number of "interesting parameters" equal to one, i.e., the template age), yields formal $68 \%$ confidence limits of $7.47 \pm 0.12 \mathrm{Myr}$ (interpolating $\chi^{2}{ }_{\nu}$ values between the $0.25 \mathrm{Myr}$ template age spacing). The secondary minimum at $14.02 \mathrm{Myr}\left(\chi_{\nu}^{2}=0.80\right)$ is statistically a worse fit at $>90 \%$ confidence. The associated $\chi^{2}$ probability for the primary minimum is 0.97 .

The two disjoint regions of low $\chi^{2}$ are due to the similarity of the SEDs at 7.5 and 14.0 Myr. Between these ages, the UV and infrared fluxes increase above the model values due to the dominance of red supergiants in the models. As mentioned above, older 

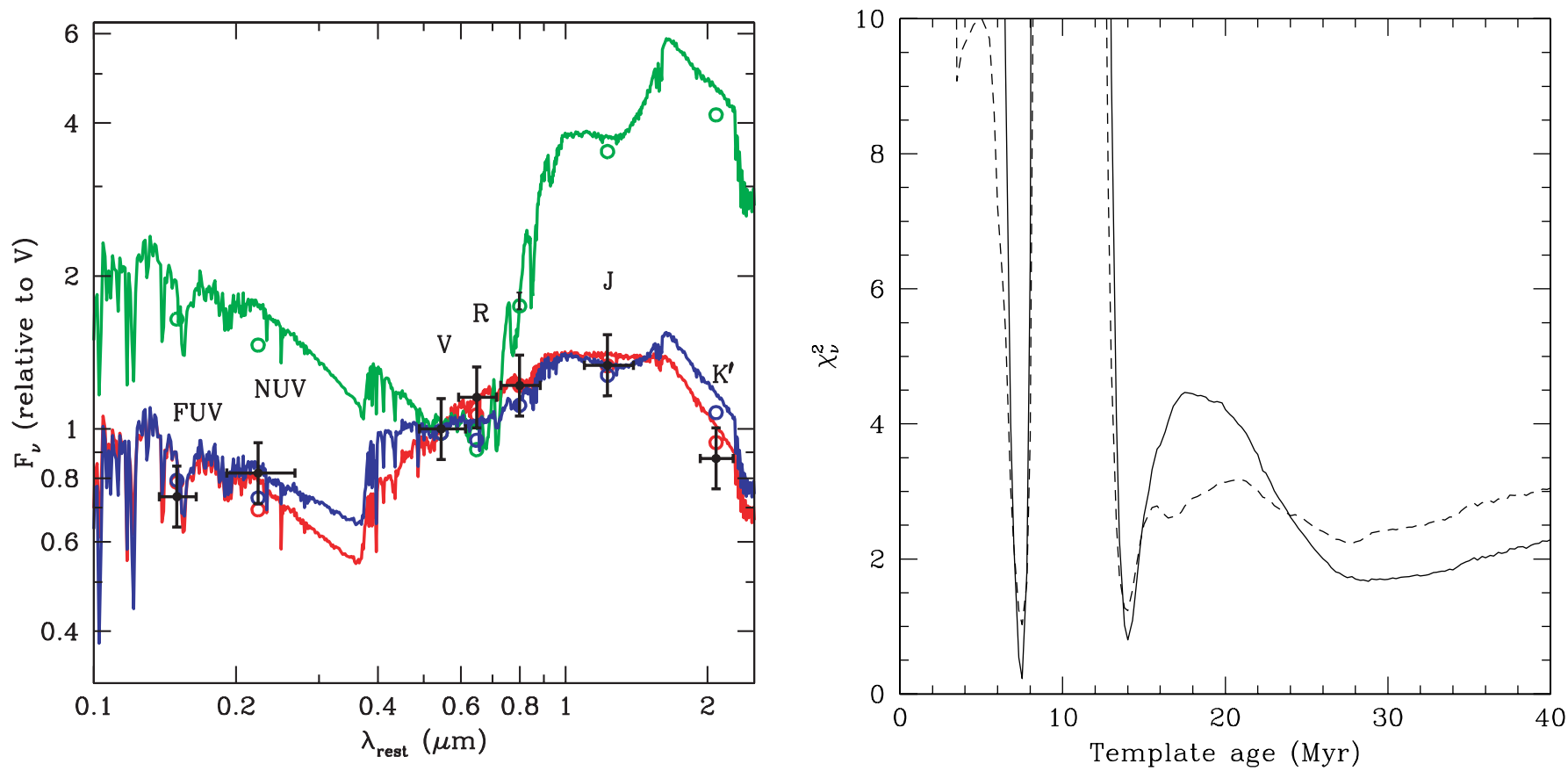

FIG. 14. - Left: Selection of synthetic spectrum models (solid lines) and the photometry computed by convolving those models with the filter transmission curves (colored open circles) compared to the observed (Galactic reddening corrected) photometry (black points). The vertical error bars on the black points represent measured photometric errors, and the horizontal bars represent the filter effective bandwidths. The best fitting ( $7.5 \mathrm{Myr}$ ) model is shown in red, along with a badly fitting model ( $9.5 \mathrm{Myr}$ in green), and another model that provides quite a good fit, i.e., low $\chi^{2}\left(14.0 \mathrm{Myr}\right.$ in blue). Right: Reduced $\chi^{2}$ for the model fits as a function of time since the instantaneous burst in the spectral template (solid line). There is a clear minimum around $7.5 \mathrm{Myr}$, and another broad, but less deep, minimum for ages older than $14 \mathrm{Myr}$. The old models fit the photometry at $V$ through $K^{\prime}$ quite well, but do a poor job of reproducing the UV flux. Adding a 1 Gyr-old population at the $40 \%$ level produces $\chi^{2}$ values shown as the dotted curve.
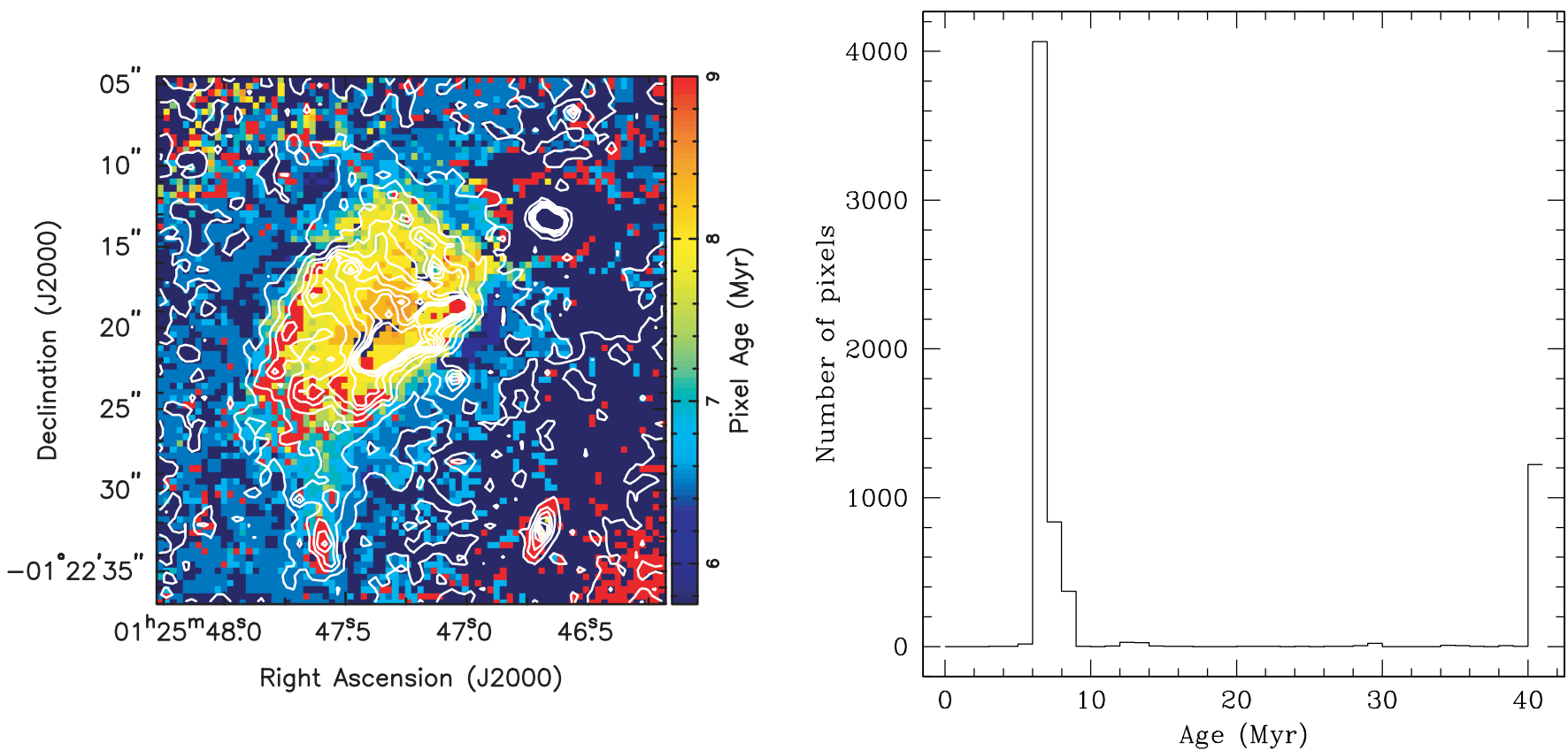

FIG. 15. - Left: Pixel age map for MO. A range in ages is seen in MO, color-coded according to the wedge at right. Dark blue pixels were rejected due to their large $\chi^{2}$ (meaning they were poorly fit). Red pixels were best fit by models of $>9 \mathrm{Myr}$ (this includes ages up to and including 1 Gyr). White contours are from the binned HST F555W image, and are overlaid to show the relationship between the morphology of $\mathrm{MO}$ and the clustering of pixels of the same age. A low $\mathrm{S} / \mathrm{N}$ results in some spurious pixels, but broadly it appears that pixels of the same age cluster together, with the oldest populations in the central bar of MO, and younger populations in the outer regions. This picture is supported by binning pixels in these regions to improve the $\mathrm{S} / \mathrm{N}$ (see text). Right: Histogram of the best fitting pixel ages. Most of the older pixels are in the nearby old elliptical NGC 541. The peak at 40 Myr includes the 1 Gyr old pixels. 
models ( $\gtrsim 25$ Myr) fit the optical and infrared points reasonably well, but do not do a good job of fitting the UV points.

In an attempt to account for an underlying (presumably old) population from the bridge and NGC 541 (see $\S 3.7$ ), we ran the $\chi^{2}$ minimization procedure again, this time adding in a $1 \mathrm{Gyr}$ population at the $40 \%$ level (the number of degrees of freedom is unchanged since the normalization of this component is fixed by the photometry from $\S 3.7)$. The best fit for the remaining component was still the $7.5 \mathrm{Myr}$ model, with $\chi_{\nu}^{2}=1.02$ (slightly higher than for the single-population model, but with a $\chi^{2}$ probability 0.41 ). This yields $68 \%$ confidence limits of $7.50_{-0.18}^{+0.17} \mathrm{Myr}$, with a secondary minimum at $13.94 \mathrm{Myr}\left(\chi_{\nu}^{2}=1.23\right)$, statistically distinct from the primary solution only at $>68 \%$ confidence.

The old population fraction was increased in steps of $1 \%$, and the $\chi^{2}$ procedure run over again. The $\chi^{2}$ minimum continued to increase (as the UV data points were less well fit), but the $7.5 \mathrm{Myr}$ model was still selected. At an old population fraction above $50 \%$, the best-fitting young population begins to fluctuate between models, and the fit becomes increasingly poor. It seems reasonable, therefore, to conclude that less than $20 \%$ (and possibly as low as $0 \%$ ) of the light (although possibly a larger percentage of the stellar mass) in the aperture is due to an old population in MO itself, as suggested by the photometry in $\S 3.7$.

We used a technique similar to that employed by Johnston et al. (2005) to obtain an age map for the galaxy. The registered, seeingconvolved images were binned $4 \times 4$ pixels. The model fitting routine, as described above, was run on the SEDs extracted from individual corresponding binned pixels in each band, producing a best-fitting age for each pixel (again assuming a single-age instantaneous starburst). The resulting pixel age map is shown in Figure 15.

Reassuringly, pixels of the same age cluster together. There appears to be a gradient in MO, from pixels with ages around 8.5 Myr at the center, to pixels with ages around 7.0 Myr at the edges. The edge of NGC 541 at the lower right of the frame, and also the nearby small background galaxy, are fit with a 1 Gyr old population (the oldest population considered in our fits). We are confident, therefore, that the pixel ages are representative of the dominant population. Since young stellar populations tend to dominate the integrated light of a galaxy, we cannot rule out an underlying old population, and indeed a small number of pixels in MO itself are best fit with older populations. However, this may be simply due to a low $\mathrm{S} / \mathrm{N}$ in the UV data in these pixels, which provides less discrimination between the primary and secondary minima in the $\chi^{2}$-age plot (the primary minimum tends to remain relatively sharply defined, whereas the secondary minimum becomes more broad, compared to the plots in Fig. 14). The pixel ages are consistent, however, with a picture in which jet-induced star formation propagates outward from a region near the central "bar" in MO.

We also extracted SEDs from the registered, convolved, binned multiwavelength images in three boxes at the locations shown in Figure 8, corresponding to MO-South, MO-North, and the filamentary structure to the north of MO. Following the same procedure as for the pixel age map, but using these larger boxes to improve the $\mathrm{S} / \mathrm{N}$, we obtain ages (with $68 \%$ confidence limits) of $6.66_{-0.31}^{+0.44}, 7.86_{-0.21}^{+0.13}$, and $7.00_{-0.33}^{+0.46} \mathrm{Myr}$, respectively for the three components. These more sensitive measurements support the conclusion from the pixel age map that MO-North was the first region to form stars, and that star formation spread over $\sim 1 \mathrm{Myr}$ to the regions further out (MO South and the NE filament have the same age to within these quoted errors). Presumably the star formation will continue to advance "downstream" in the future, as the gas seen in the $\mathrm{H}$ I map begins to form stars.

\section{DISCUSSION}

Our new observations of MO reinforce the conclusion by van Breugel et al. (1985) that MO is a young starburst triggered by the radio jet from NGC 541. However, some interesting questions remain; first, what existed at that location before the jet entered? Possibilities range from a preexisting gas-rich galaxy or a cold gas cloud, to a warm or even hot diffuse cloud of gas. Second, what are the physical conditions in the jet (speed, Mach number, mass loading, etc.) such that it is able to trigger this starburst? Our observations add some further insight to the first question. To learn more about the second will probably first require a better understanding of FR I jets in general (Young et al. 2005; Eilek et al. 2002; Kataoka et al. 2006) and, ultimately, numerical simulations of these types of jets.

\subsection{Kinematics and Morphology}

The neutral hydrogen emission is intimately associated with the radio jet: the H I appears to be "wrapped around" the axis of the jet, and there is no $\mathrm{H}$ i outside the jet. It will be important to search for possible very low surface brightness $\mathrm{H}$ I that might exist near MO, in the bridge or elsewhere, since our observations were taken in the $\mathrm{C}$ array, and very large-scale emission might have been missed. But given the current observational evidence, there is good reason to believe that there was no extended $\mathrm{H} \mathrm{I}$ prior to the jet interaction, and therefore that the $\mathrm{H}$ i is entirely the result of radiative cooling of warmer gas in the intergalactic medium triggered by the shocks induced in it by the passage of the radio jet.

Numerical simulations of jet-cloud collisions, subject to assumptions about the radio jet and the multiphase medium with which it interacts, show that radiative cooling may indeed lead to star formation (Fragile et al. 2004). The mass inferred for the $\mathrm{H}_{\mathrm{I}}$ cloud is $4.9 \times 10^{8} M_{\odot}$, and the projected extent of the $\mathrm{H}$ I emission is $275 \mathrm{kpc}^{2}$ ( 2.7 ). The total amount of (hot) gas in this area according to the observations of Nikogossyan et al. (1999) is expected to be $\sim 1.4 \times 10^{9} M_{\odot}$, so the available reservoir is certainly large enough. The question is: how did it cool to form the $\mathrm{H}$ i? Until we have better X-ray data, we will have to assume that the IGM in the Abell 194 cluster resembles to some extent that of so-called cooling flow clusters, which contain both hot $\left(10^{6}-10^{7} \mathrm{~K}\right)$ and cool/warm $\left(10^{4} \mathrm{~K}\right)$ gas. We note that Abell 194 is reportedly a "poor and cold" cluster (Nikogossyan et al. 1999), and as such, a population of relatively cool, dense clouds in its IGM may well exist.

The jet-H I relationship in MO is different from that in Centaurus $\mathrm{A}$, where the jet is running into a preexisting, very extended $\mathrm{H}$ i structure found in rotation around NGC 5128 (Schiminovich et al. 1994). Another difference is that in MO the $\mathrm{H}$ I is downstream and the star formation is closer to the AGN, while in Cen $\mathrm{A}$ it is the other way around: the $\mathrm{H}_{\mathrm{I}}$ is closest to the AGN and the star formation is downstream. Perhaps this is the difference between a jet hitting an existing $\mathrm{H}_{\mathrm{I}}$ cloud (Cen A) and the jet causing the warm gas to cool to form $\mathrm{H}_{\mathrm{I}}$ (MO).

The $\mathrm{H}$ i shows a significant velocity gradient (up to $100 \mathrm{~km} \mathrm{~s}^{-1}$ ) at the jet-cloud interaction site (Oosterloo \& Morganti 2005). Other examples of even larger $\mathrm{H}$ I outflows $\left(1000 \mathrm{~km} \mathrm{~s}^{-1}\right)$ have recently been discovered in a number of radio galaxies, to the extent that these may remove significant amounts of gas from their parent galaxies (Morganti et al. 2005). However, these are all very luminous far-infrared galaxies with lots of ISM cold gas and dust through which the jets are propagating. In these cases, it is reasonable to assume that the $\mathrm{H}$ I gas may have been entrained in some way, as it may also have in Cen A (Oosterloo \& Morganti 2005). 
In MO the velocity gradient is much smaller, $\sim 40 \mathrm{~km} \mathrm{~s}^{-1}$, but the scenario may be qualitatively the same, although in MO the entrained gas clouds were probably not cold but warm. However, in this case the clouds will also be accelerated, while they cool radiatively. Following Fragile et al. (2004), the velocity $v_{\mathrm{cl}}$ of the accelerated clouds is

$$
\frac{v_{\mathrm{cl}}}{\mathrm{km} \mathrm{s}^{-1}} \lesssim 20\left(\frac{\chi}{10^{3}}\right)^{-1}\left(\frac{R_{\mathrm{cl}}}{100 \mathrm{pc}}\right)^{-1}\left(\frac{v_{\mathrm{sh}, b}}{10^{3} \mathrm{~km} \mathrm{~s}^{-1}}\right)^{2}\left(\frac{t}{\mathrm{Myr}}\right)
$$

where $\chi=n_{\mathrm{cl}} / n_{b, i}$ is the density contrast between the cloud and background intercloud medium, $R_{\mathrm{cl}}$ is the cloud radius, $v_{\mathrm{sh}, b}$ is the shock velocity in the background intercloud medium, and $t$ is the time during which the cloud is being accelerated. This time will be at most $t_{\mathrm{acc}} \sim \sqrt{\chi} t_{\mathrm{cc}} \mathrm{yr}$, where $t_{\mathrm{cc}}$ is the cloud crossing time for the radiative shocks. For the parameters used by Fragile et al. one finds $t_{\mathrm{cc}} \sim 1 \mathrm{Myr}, t_{\mathrm{acc}}<30 \mathrm{Myr}$, and $v_{\mathrm{cl}} \sim 10-100 \mathrm{~km} \mathrm{~s}^{-1}$. Thus within the assumed framework of a jet interacting with a clumpy, warm medium, it is possible to explain the observed $\mathrm{H}$ I velocity shear of $\sim 40 \mathrm{~km} \mathrm{~s}^{-1}$.

The VLA H I, GALEXUV, and ATT H $\alpha$ images all reveal that $\mathrm{MO}$ has a double structure that straddles the radio jet. In fact, the overall UV appearance of MO now looks very much like the radio-aligned rest-frame $\mathrm{UV}$ structure seen in the $z=3.8$ radio galaxy 4C 41.17 (van Breugel et al. 1999), which is also thought to be due to jet-induced star formation (Dey et al. 1997; Bicknell et al. 2000). This is an important qualitative result because it seems now more appropriate to assume that the star formation in $\mathrm{MO}$ occurs in the shear layers at the wall of the jet, i.e., not in a head-on jet-cloud collision. This means that the jet-cloud collision in MO may be a more complex astrophysical problem than initially thought.

The jet speed in such a shear layer will be lower than at the center, due to jet expansion and entrainment (Laing \& Bridle 2002). The pressure in the wall shock is also lower than in the bow shock of the jet. This translates to a lower speed for the radiative shocks in the entrained clouds, and the parameter space studied by Fragile et al. (2004) then indicates that star formation may be easier to accomplish, at least with a certain range of preshock cloud densities. But perhaps more important is that shear layers of FR I type jets are active sites of relativistic particle acceleration, as evinced by radio spectral tomographic imaging, which shows a remarkably flat radio spectral energy distribution across the jets (Young et al. 2005). This is further supported by recent $X$-ray observations of the nearest FR I radio jet in Centaurus A (Kataoka et al. 2006), which show that the jet is edge brightened with a possible hardening of the X-ray spectrum in the outer regions. As a result, the pressure of the relativistic fluid in these regions will be enhanced, which may affect the interaction with the clouds and the star formation process.

The peak $\mathrm{H}$ I column densities in the $\mathrm{H}$ I blobs are about $N_{\mathrm{H}_{\mathrm{I}}}=4.5 \times 10^{20} \mathrm{~cm}^{-2}$. This is just at the bottom of the range where, under galactic conditions, star formation would occur (Schaye 2004), but considering the relatively low spatial resolution of the $\mathrm{H}$ I data, it could match more or less. However, the observed star formation sites are several kpc away from where the peaks in the $\mathrm{H}$ I data are, i.e., the star formation is not happening where the H I says it should happen. So it may not be obvious to make the usual link between star formation and $\mathrm{H}$ I column density. The same is true in the $\mathrm{H}$ I filament near Centaurus A, where the $\mathrm{H}$ I column densities are well above $10^{21} \mathrm{~cm}^{-2}$, but only at those locations where there is no star formation.
One can also compare the global star formation efficiency $M_{\text {stars }} / M_{\mathrm{H}}$ in $\mathrm{MO}$ to that of other systems. For MO we find $\sim 4 \%$, which is similar to that in Centaurus A (a few percent; Oosterloo \& Morganti 2005), and indeed typical for starbursts in isolated dwarf galaxies (Fritze-v. Alvensleben 2005, and references therein).

It is puzzling that, while the $\mathrm{H}$ I emission downstream from MO-South is comparable to that of MO-North, the $\mathrm{H} \alpha$ and UV emission, and hence the current star formation activity, is much less there. It should be noted in this regard that the jet is disrupted in the region of MO, so it may well be that the jet-cloud interaction is simply stronger in MO-North than in MO-South. This would be the case if, for example, the sense of the jet precession was toward the north.

The HST images for the first time reveal the presence of numerous "blobs," which appear to be young clusters associated with $\mathrm{H}$ II regions. We extracted photometry in 0 ". 5 -radius apertures for 20 of these blobs (Fig. 13), converting from the HST filters to Johnson $V$ and $I$, and transforming the $V$-band measurements to absolute magnitudes. Comparison with van den Bergh (1995) shows that the absolute magnitudes are what would be expected of young star clusters, and the colors of these blobs, when compared to Figure 11 of Whitmore \& Schweizer (1995), are just what would be expected for ages of a few Myr (consistent with all our other data). $U$-band data would be necessary in order to pin down these ages more accurately, and we would need to reach fainter magnitudes in order to compare with the color-magnitude diagrams for individual stars in Centaurus A by Mould et al. (2000) .

\subsection{Timing}

Due to the tendency of a young population to dominate the integrated starlight in a galaxy, it is difficult to completely rule out the presence of an older preexisting population. The situation in $\mathrm{MO}$ is even more complex due to its location in the outskirts of the elliptical galaxy NGC 541, and in the bridge of stars connecting NGC 541 with two of the other ellipticals in the cluster. Certainly, the spectroscopic and photometric data presented here agree that a population of order 7.5 Myr old is present in MO, and dominates the light output. The consistency of the pixel ages, the low internal reddening, and the lack of absorption lines expected from an old population also support the conjecture that MO consists of a single-age population. In addition, the similar morphologies at $\mathrm{F} 555 \mathrm{~W}$ and $K^{\prime}$ are not what would be expected if an old, gas-rich dwarf galaxy wandered into the jet and had a new burst of star formation "switched on" by the jet-cloud interaction. The star formation also appears at the leading edge of the $\mathrm{H}$ I cloud, as would be expected if it was the jet that caused the $\mathrm{H}$ I and subsequently the stars to form (since it takes time for the stars to form after the passage of the jet). As discussed above, however, it is not possible using these data to completely rule out the presence of an older underlying population in MO. It does seem clear, however, that the picture of a jet-cloud interaction, sometime a little more than 7.5 Myr ago, would be capable of causing the observed phenomena.

Examination of the radio maps shows that a jet plume (van Breugel et al. 1985) extends up to $37 \mathrm{kpc}$ "downstream" of MO (or somewhat further if the jet is not in the plane of the sky). It seems unlikely that this is a buoyant bubble (Brüggen \& Kaiser 2001), as the jet is still "switched on" and the plume does not display the characteristic "mushroom cloud" shape. The fact that the jet is well collimated until its interaction with MO, and disrupted downstream, as well as the similar size of the $\mathrm{H}$ I cloud to the width of the jet, suggest that as the jet expanded, it ran into the gas cloud that ultimately formed MO. If MO was a preexisting 
galaxy that wandered into the jet, it is unlikely that it would have done so at the precise time to cause the decollimation seen.

The age of the downstream cocoon can therefore be estimated as the age of MO, plus the time taken for stars to begin forming after the jet-cloud interaction. Taking a typical star formation timescale of $\lesssim 1 \mathrm{Myr}$ (Fragile et al. 2004), we assume that the cocoon expanded by at least $37 \mathrm{kpc}$ in $8.5 \mathrm{Myr}$, i.e., at $\gtrsim 4 \times 10^{3} \mathrm{~km} \mathrm{~s}^{-1}$.

If we assume that the SFR has been roughly constant during the lifetime of $\mathrm{MO}$, this yields a timescale for the formation of the $1.9 \times 10^{7} M_{\odot}$ of stars of $36.5 \mathrm{Myr}$. This is only a few times the 7.5 Myr inferred from the SED age dating. Since it is plausible that the SFR was somewhat higher in the past, it is reasonable to assume that all the stars may have been formed since the jet-cloud interaction. The timescale for depletion of the observed $\mathrm{H} \mathrm{I}$ is much longer $(\sim 1 \mathrm{Gyr})$.

\section{CONCLUSIONS}

We have presented new evidence that the star formation in MO was induced by a radio jet, primarily in the outer shear layers. This is similar to the jet-induced star formation associated with the Centaurus A jet, and the radio-aligned star-forming regions in powerful radio galaxies at high redshift. Absorption and emission-line measurements, and broadband SED fitting give a consistent age of around 7.5 Myr for MO. While it is not possible to completely rule out the presence of an old population in MO, the data are consistent with MO having formed de novo when the jet interacted with the ambient ISM/IGM. We discovered a double neutral hydrogen cloud that straddles the radio jet downstream of MO, at a location where the jet changes direction and decollimates. Unlike Centaurus A, we propose that the $\mathrm{H}$ I associated with $\mathrm{MO}$ formed in situ through cooling of clumpy, warm gas, in the stellar bridge or cluster IGM, as it was compressed by radiative shocks at the jet collision site, in agreement with earlier numerical simulations (Fragile et al. 2004). The star formation in $\mathrm{MO}$ then followed from the cooling and collapse of such $\mathrm{H} \mathrm{I}$ clouds, and the $\mathrm{H}$ I kinematics, which show $40 \mathrm{~km} \mathrm{~s}^{-1}$ shear velocities, are also consistent with such models. The FR I type radio source associated with $\mathrm{MO}$ is orders of magnitude lower in radio luminosity, and correspondingly more common, than the very luminous FR II type radio galaxies and their aligned star-forming regions at high redshift. Nevertheless, as MO shows, these types of radio sources can still trigger star formation, even if the ambient medium is only moderately dense. This suggests that jet-induced star formation may have been quite common in the early universe, where gas densities in their parent, forming galaxies and surrounding protoclusters were much higher than today, and when AGN activity was much more prevalent.

We thank the staff of the Lick and Siding Spring Observatories for their support. Thanks to Michael Gregg for providing us with Lick PFCam data. We thank the anonymous referee for helpful and constructive suggestions. Data presented here were obtained at the W. M. Keck Observatory, which is operated as a scientific partnership among the California Institute of Technology, the University of California, and the National Aeronautics and Space Administration. The Observatory was made possible by the generous financial support of the W. M. Keck Foundation. Work was also based on observations made with the NASA/ESA Hubble Space Telescope, obtained from the data archive at the Space Telescope Science Institute. STScI is operated by the Association of Universities for Research in Astronomy, Inc. under NASA contract NAS 5-26555. GALEX (Galaxy Evolution Explorer) is a NASA Small Explorer, launched in 2003 April. We gratefully acknowledge NASA's support for construction, operation, and science analysis for the GALEX mission, developed in cooperation with the Centre National d'Etudes Spatiales of France and the Korean Ministry of Science and Technology. The National Radio Astronomy Observatory is a facility of the National Science Foundation operated under cooperative agreement by Associated Universities, Inc. Some of the data presented in this paper were obtained from the Multimission Archive at the Space Telescope Science Institute (MAST). Support for MAST for non-HST data is provided by the NASA Office of Space Science via grant NAG5-7584 and by other grants and contracts. Dopita acknowledges the support of both the Australian National University and the Australian Research Council (ARC) through his ARC Australian Federation Fellowship, and financial support through ARC Discovery project grant DP0208445. Work was performed under the auspices of the US Department of Energy, National Nuclear Security Administration by the University of California, Lawrence Livermore National Laboratory under contract W-7405-Eng-48. S. C. and W.v. B. acknowledge support for radio galaxy studies at the University of California, Merced, including the work reported here, with the Hubble, Spitzer and Chandra space telescopes via NASA grants HST 10127, SST 3482, SST 3329, and Chandra/CXO 06701011.
Anninos, P., Fragile, P. C., \& Murray, S. D. 2003, ApJS, 147, 177

Avni, Y. 1976, ApJ, 210, 642

Bicknell, G. V., Sutherland, R. S., van Breugel, W. J. M., Dopita, M. A., Dey, A., \& Miley, G. K. 2000, ApJ, 540, 678

Böker, T., et al. 1999, ApJS, 124, 95

Brodie, J., Koenigl, A., \& Bowyer, S. 1983, ApJ, 273, 154

Brüggen, M., \& Kaiser, C. R. 2001, MNRAS, 325, 676

Calzetti, D. 2001, NewA Rev., 45, 601

Calzetti, D., Kinney, A. L., \& Storchi-Bergmann, T. 1994, ApJ, 429, 582

de Robertis, M. M., Dufour, R. J., \& Hunt, R. W. 1987, JRASC, 81, 195

Dey, A., van Breugel, W., Vacca, W. D., \& Antonucci, R. 1997, ApJ, 490, 698

De Young, D. S. 1989, ApJ, 342, L59

Dopita, M. A., Binette, L., Dodorico, S., \& Benvenuti, P. 1984, ApJ, 276, 653

Dopita, M. A., Groves, B. A., Sutherland, R. S., Binette, L., \& Cecil, G. 2002, ApJ, 572, 753

Dopita, M. A., \& Hua, C. T. 1997, ApJS, 108, 515

Dopita, M. A., Kewley, L. J., Heisler, C. A., \& Sutherland, R. S. 2000, ApJ, 542,224

Dopita, M. A., \& Ryder,S. D. 1994, ApJ, 430, 163

Dopita, M. A., \& Sutherland, R. S. 1995, ApJ, 455, 468
REFERENCES

Eilek, J., Hardee, P., Markovic, T., Ledlow, M., \& Owen, F. 2002, NewA Rev., 46,327

Fanaroff, B. L., \& Riley, J. M. 1974, MNRAS, 167, 31P

Ferland, G. J., Fabian, A. C., \& Johnstone, R. M. 2002, MNRAS, 333, 876 Filippenko, A. V. 1982, PASP, 94, 715

Fragile, P. C., Murray, S. D., Anninos, P., \& van Breugel, W. 2004, ApJ, 604, 74 Fritze-v. Alvensleben, U. 2005, preprint (astro-ph/0508099)

Gil de Paz, A., et al. 2006, ApJS, in press (astro-ph/0606440)

González Delgado, R. M., Leitherer, C., \& Heckman, T. M. 1999, ApJS, 125, 489

Graham, J. A., \& Price, R. M. 1981, ApJ, 247, 813

Johnston, H. M., Hunstead, R. W., Cotter, G., \& Sadler, E. M. 2005, MNRAS, 356,515

Kataoka, J., Stawarz, L., Aharonian, F., Takahara, F., Ostrowski, M., \& and

Edwards, P. G. 2006, ApJ, 641, 158

Kennicutt, R. C. 1998, ARA\&A, 36, 189

Kewley, L. J., \& Dopita, M. A. 2002, ApJS, 142, 35

Klamer, I. J., Ekers, R. D., Sadler, E. M., \& Hunstead, R. W. 2004, ApJ, 612, L97

Laing, R. A., \& Bridle, A. H. 2002, MNRAS, 336, 1161

Leitherer, C., et al. 1999, ApJS, 123, 3

Martin, D. C., et al. 2005, ApJ, 619, L1 
McNamara, B. R. 2002, NewA Rev., 46, 141

Minkowski, R. 1958, PASP, 70, 143

Morganti, R., Tadhunter, C. N., \& Oosterloo, T. A. 2005, A\&A, 444, L9

Morrissey, P., et al. 2005, ApJ, 619, L7

Mould, J. R., et al. 2000, ApJ, 536, 266

Nikogossyan, E., Durret, F., Gerbal, D., \& Magnard, F. 1999, A\&A, 349, 97

O’Dea, C. P., Baum, S. A., Mack, J., Koekemoer, A. M., \& Laor, A. 2004, ApJ, 612,131

Oke, J. B., et al. 1995, PASP, 107, 375

Oosterloo, T. A., \& Morganti, R. 2005, A\&A, 429, 469

Panuzzo, P., Bressan, A., Granato, G. L., Silva, L., \& Danese, L. 2003, A\&A, 409, 99

Rees, M. J. 1989, MNRAS, 239, 1P

Schaye, J. 2004, ApJ, 609, 667

Schiminovich, D., van Gorkom, J. H., van der Hulst, J. M., \& Kasow, S. 1994, ApJ, 423, L101

Schlegel, D. J., Finkbeiner, D. P., \& Davis, M. 1998, ApJ, 500, 525

Sheinis, A. I., et al. 2002, PASP, 114, 851

Simkin, S. M. 1976, ApJ, 204, 25
Smith, R. J., Lucey, J. R., Hudson, M. J., Schlegel, D. J., \& Davies, R. L. 2000, MNRAS, 313, 469

Spergel, D. N., et al. 2003, ApJS, 148, 175

Stasinska, G., \& Leitherer, C. 1996, ApJS, 107, 661

Sutherland, R. S., \& Dopita, M. A. 1993, ApJS, 88, 253

Trager, S. C., Faber, S. M., Worthey, G., \& González, J. J. 2000, AJ, 119, 1645 van Breugel, W., Filippenko, A. V., Heckman, T., \& Miley, G. 1985, ApJ, 293, 83

van Breugel, W., et al. 1999, in Proc. of the Colloq., The Most Distant Radio Galaxies, ed. H. J. A. Röttgering, P. N. Best, \& M. D. Lehnert (Amsterdam: Royal Netherlands Acad. of Arts and Sci.), 49

van den Bergh, S. 1995, Nature, 374, 215

Veilleux, S., \& Osterbrock, D. E. 1987, ApJS, 63, 295

Verdoes Kleijn, G. A., Baum, S. A., de Zeeuw, P. T., \& O’Dea, C. P. 1999, AJ, 118,2592

Whitmore, B. C., \& Schweizer, F. 1995, AJ, 109, 960

Wills, K. A., Morganti, R., Tadhunter, C. N., Robinson, T. G., \& Villar-Martin, M. 2004, MNRAS, 347, 771

Young, A., Rudnick, L., Katz, D., DeLaney, T., Kassim, N. E., \& Makishima, K. 2005, ApJ, 626, 748 